\title{
Theory of Dilute Binary Granular Gas Mixtures
}

\author{
D. Serero ${ }^{1}$, S. H. Noskowicz and I. Goldhirsch \\ School of Mechanical Engineering, \\ Faculty of Engineering, Tel Aviv University, Ramat-Aviv, Tel Aviv 69978, Israel
}

\begin{abstract}
A computer-aided method for accurately carrying out the Chapman-Enskog expansion of the Boltzmann equation, including its inelastic variant, is presented and employed to derive a hydrodynamic description of a dilute binary mixture of smooth inelastic spheres. Constitutive relations, formally valid for all physical values of the coefficients of restitution, are calculated by carrying out the pertinent Chapman-Enskog expansion to sufficient high orders in the Sonine polynomials to ensure numerical convergence. The resulting hydrodynamic description is applied to the analysis of a vertically vibrated binary mixture of particles (under gravity) differing only in their respective coefficients of restitution. It is shown that even with this "minor" difference the mixture partly segregates, its steady state exhibiting a sandwich-like configuration.
\end{abstract}

Key words: kinetic theory, granular gases, mixtures, segregation, Chapman-Enskog expansion, hydrodynamics

AMS subject classification: 76P05, 82B40, 82C40, 82C70

\section{Introduction}

Granular materials, i.e. collections of macroscopic solid grains, are ubiquitous in Nature and of central importance in industry. They exhibit a fascinating and often counter-intuitive range of phenomena, behaving at times like solids (when at rest), liquids, or gases [17, 22]. The diversity of behavior they exhibit can be attributed in part to the fact that the grain interactions are dissipative [17]. The typical polydispersity of grains in Nature and industry is yet another source for the rich range of phenomena exhibited by these materials. Indeed, granular mixtures exhibit a host of

\footnotetext{
${ }^{1}$ Corresponding author. E-mail: serero@eng.tau.ac.il
} 
effects that are specific to them, one of the most fascinating (and important) undoubtedly being their tendency to spontaneously segregate under external forcing [33, 46, 29]. They may segregate even as a result of small differences in the properties of their constituents, such as mass, shape, frictional properties $[28,48]$ or coefficients of restitution $[43,6]$. Segregation may appear as a result of a phase transition [37], or be driven by time-dependent external means, such as ratcheting $[12,36]$; in some case the segregated states are the only ones "allowed", e.g., in shear flows or vibrated systems.

An intriguing and famous example of (size) segregation in vertically vibrated systems is the 'Brazil nut' (BN) effect, whereby a relatively large particle in a vibrated granular system tends to climb to the 'top' of the system, against gravity [38], almost irrespective of its mass. An opposite phenomenon, the "Reverse Brazil Nut" effect (RBN), has been recently observed and discussed [20] as well. Several explanations of these phenomena (or more generally, granular segregation in vertically vibrated systems) have been proposed [26, 45, 47, 2, 31], invoking a variety of mechanisms whose relevance varies with the nature of the system and the type of forcing it is subject to [39]. For weak excitations (i.e. relatively low energy input), void filling [38, 29] seems to be among the prominent mechanisms of size separation. This is basically a geometrical effect: during the compaction phase of a shaking cycle a small particle is more likely to find a void in the layer below it than a larger particle, resulting in the accumulation of larger particles at the top of the system. Another important mechanism of size segregation is provided by the action of convection rolls [27, 26, 35] in vertically shaken systems; these are triggered by the friction between the grains and the container walls but their dynamics parallels that of the rolls in Rayleigh-Bénard convection. Consider a system of aspect ratio between 1 and 2: the velocity field associated with the rolls points upward in the center of the system and downward at the sidewalls of the container; larger particles, lifted by the central upstream, may be trapped at the top of the system when their sizes prevent them from joining the downward flow near the walls [38, 29, 26, 27, 9]. When these wall layers are sufficiently wide the large particles can move inside them; in this case convection gives rise to mixing $[45,7,39]$.

When a granular system is vigorously shaken it becomes a "granular gas" in which the grain interaction mechanism is binary near-instantaneous collisions. In this case, kinetic theory, properly modified to account for the inelasticity of the collisions, is expected to provide a reliable description of the kinetics and hydrodynamics of the system, and in particular account for segregation in mixtures. This is indeed the case.

Since the Brazil nut effect and segregation in granular mixtures in general occur even in the absence of convection and when the mechanism of void filling is irrelevant, one may wonder as to the origin of this phenomenon. It turns out that the Soret phenomenon or its single-particle manifestation, thermophoresis, is always active in vertically vibrated granular systems. Since the system is dissipative and energy is pumped into it at the (vibrating) floor, the kinetic energy of the grains (or granular temperature) decays away from the source of energy, i.e. as a function of the distance from the floor. This gives rise to a granular temperature gradient. Thermophoresis forces large or massive particles to move down temperature gradients $[18,19]$ and thus segregate. We reiterate that the conditions for thermophoresis are created by the inelasticity of the grains, i.e. segregation, in particular the Brazil nut effect, is an indirect result of inelasticity [21]. Experimental evidence 
for the latter mechanism in the $\mathrm{BN}$ effect for highly agitated systems has recently been reported [39]. Arnarson and Willits [1] considered the thermal diffusion factor to study size segregation in the presence or the absence of gravity. However, their theory, which improves upon the results of Jenkins and Mancini [23] differs from the elastic theory of [25] only in the fact that it includes a sink term in the equation for the temperature (representing the energy loss due to collisions), and therefore no other inelastic effects (e.g., on the transport coefficients) are accounted for in their theory of thermal diffusion. Recently, a theory for granular mixtures [13, 14] has been worked out to study the transition between the $\mathrm{BN}$ and $\mathrm{RBN}$ effects (it also treats finite concentrations of intruders). Slightly different approaches, still based on kinetic theory, have been invoked to obtain a segregation criterion $[24,51,47]$. These approaches consider the temperature gradient as input, and therefore only partly address the case of a vibrated system; they may capture though some of its qualitative segregation properties for known temperature profiles.

The above description of the temperature profile for a vertically vibrated granular system is somewhat inaccurate. Theoretical studies, most of which focus on the monodisperse case, as well as numerical simulations, reveal that after the initial decrease in the value of the granular temperature as a function of height above the floor, the temperature profile possesses a minimum above which the temperature increases as a function of height $[3,49,43]$. Therefore thermophoresis would push large or massive intruders to this minimum rather than the top of the system. If the system is sufficiently small the minimum may not be reached or be close to the top (note: the top of a vibrated dilute granular system is diffuse, i.e. rather ill defined; in practice the top is taken to be the maximal height were one can still collect statistics, i.e. the volume fraction is larger than say 0.001 ) in which case intruders will move to the top. The problem of a single intruder in a vibrated granular gas was treated in [4], and a comparison between theory, experiments and simulations for slightly inelastic binary mixtures was given in [50]. Recently, it was shown [43] that a dilute vertically vibrated binary mixture of particles of the same size with different masses arranges itself in a sandwich-like configuration in which a layer of heavy particles is trapped between two layer of light particles. This is a result of the competition between buoyancy and thermal diffusion: the former pushes the light particles to the top and the latter pushes the heavy particles to the region of minimal temperature, leaving room for the light particles (also) at the bottom of the system. While in most of these studies the segregation mechanisms involved are directly or indirectly related to the inelastic nature of the granular constituents, few studies have concentrated on the direct influence of inelasticity alone on segregation, which would be the purest manifestation of the effect of inelasticity. In reference [43] it was shown that for near-elastic collisions particles with identical mass and size may segregate on the basis of differences in their inelastic properties alone, when subject to a temperature gradient. This was corroborated in molecular dynamic (MD) simulations of vertically vibrated granular gas mixtures $[43,6]$. Here the analysis of [43] is extended to finite degrees of inelasticity and the problem of vertically vibrated mixtures of particles differing only in their respective coefficients of normal restitution is addressed.

The structure of this paper is as follows. Section 2. provides a description of the system studied below, basic definitions, general continuum mechanical results and a formulation of the considered problem. Section 3. provides the kinetic formulation of the problem, describes the novel computeraided method we employ for the analysis of the kinetic problem and presents the form of the 
constitutive relations. Section 4. presents an application of the results to the problem of inelasticity induced segregation. Finally, Section 5. provides concluding comments. Some technical details are relegated to a series of Appendices.

\section{Definition of the system and its description}

We consider a binary mixture of smooth hard spheres, comprising species $A$ and $B$, of masses $m_{A}$ and $m_{B}$, and diameters $\sigma_{A}$ and $\sigma_{B}$, respectively. The coefficient of normal restitution (assumed to be fixed) for a collision of a particle of species $\alpha \in\{A, B\}$ with a particle of species $\beta \in\{A, B\}$ is denoted by $e_{\alpha \beta}$ (hence, there are three possibilities: $e_{A A}, e_{A B}$ and $e_{B B}$ ). Tangential (frictional) restitution is not considered here. The transformation of velocities due to a collision of a sphere of species $\alpha$ with a sphere of species $\beta$ is given by [43]:

$$
\begin{aligned}
& \mathbf{v}_{1}=\mathbf{v}_{1}^{\prime}-\left(1+e_{\alpha \beta}\right) M^{\beta \alpha}\left(\mathbf{v}_{12}^{\prime} \cdot \hat{\mathbf{k}}\right) \hat{\mathbf{k}} \\
& \mathbf{v}_{2}=\mathbf{v}_{2}^{\prime}+\left(1+e_{\alpha \beta}\right) M^{\alpha \beta}\left(\mathbf{v}_{12}^{\prime} \cdot \hat{\mathbf{k}}\right) \hat{\mathbf{k}}
\end{aligned}
$$

where $\left\{\mathbf{v}_{1}^{\prime}, \mathbf{v}_{2}^{\prime}\right\}$ denote the precollisional velocities of the spheres (the index ' 1 ' refers here to species $\alpha$ ) and $\left\{\mathbf{v}_{1}, \mathbf{v}_{2}\right\}$ the corresponding postcollisional velocities; $\hat{\mathbf{k}}$ is a unit vector pointing from the center of sphere $\alpha$ to that of sphere $\beta, M^{\alpha \beta} \equiv \frac{m_{\alpha}}{m_{\alpha}+m_{\beta}}$, and $\mathbf{v}_{12} \equiv \mathbf{v}_{1}-\mathbf{v}_{2}$ (a similar definition holding for the primed velocities). Obvious kinematic constraints require that $\mathbf{v}_{12} \cdot \hat{\mathbf{k}} \geq 0$. It is convenient to define the degrees of inelasticity, corresponding to the coefficients of restitution, as: $\varepsilon_{\alpha \beta} \equiv 1-e_{\alpha \beta}^{2}$. In the realm of molecular (or elastically colliding) gases, the hydrodynamic fields are the densities of the conserved fields. In the case of a binary mixtures, these consist of the two number densities, $n_{A}$ and $n_{B}$ (or the corresponding mass densities $\rho_{A}=n_{A} m_{A}$ and $\rho_{B}=n_{B} m_{B}$ ), the mixture's velocity field, $\mathbf{V}$ (or momentum density), which is defined as a mass average of the species' mean velocities, and the temperature field, $T$, defined as the mean fluctuating kinetic energy of a fluid particle). Although energy is not a conserved entity for inelastic (granular) gases, it is still included in the set of the hydrodynamic fields, as it characterizes the (important) particle velocity fluctuations.

\subsection{Continuum mechanical equations of motion}

The form of the equations of motion for the hydrodynamic fields follows from the conservation laws, Galilean invariance and standard tensorial considerations, and as such they are very general (e.g., not limited to dilute gases). They also follow from the Boltzmann equation directly (using the standard procedure of multiplying the Boltzmann equation by products of the particle velocity components, followed by integration over the velocities; see more below).

The equation of motion for the number density, $n_{\alpha}$, with $\alpha \in\{A, B\}$, is:

$$
\frac{D n_{\alpha}}{D t}=-\operatorname{div} \mathbf{J}_{\alpha}-n_{\alpha} \operatorname{div} \mathbf{V}
$$


where $\frac{D}{D t}=\frac{\partial}{\partial t}+\mathbf{V} \cdot \nabla$ is the material derivative and

$$
\mathbf{J}_{\alpha}=n_{\alpha}\left(\mathbf{V}_{\alpha}-\mathbf{V}\right)
$$

is the particle flux density of species $\alpha$. As $\mathbf{V}_{\alpha}$, the velocity field of species $\alpha$, or equivalently, the flux, $\mathbf{J}_{\alpha}$, is not a hydrodynamic field, it must be given by an appropriate constitutive relation. To Navier-Stokes order (linear in the gradients of the hydrodynamic fields) one can write this entity as:

$$
\mathbf{J}_{\alpha}=\frac{\sqrt{6}}{6} \frac{1}{\sigma_{A B}^{2}} \frac{n_{\alpha}}{n} \sqrt{\frac{T}{m_{\alpha}}}\left(\kappa_{\alpha}^{T} \nabla \ln T+\kappa_{\alpha}^{n} \nabla \ln n+\kappa_{\alpha}^{c} \nabla \ln c\right),
$$

where $n \equiv n_{A}+n_{B}$ is the total number density of the mixture, $c \equiv \frac{n_{A}}{n}$ is the concentration of $A$ particles, and the transport coefficients $\kappa_{\alpha}^{T}, \kappa_{\alpha}^{n}$ and $\kappa_{\alpha}^{c}$ depend on the parameters characterizing the particles (masses, diameters and coefficients of restitution) and the concentration field, $c$. The prefactor assures that the transport coefficients are dimensionless and the numbers appearing in it are there for technical convenience, the same comment holding for the expression for the heat flux given below (see below concerning the expansions of these transport coefficients). As the Boltzmann equation produces an equation of state which is the same as that for an ideal gas, the pressure is given (with the normalization of the temperature defined below) by: $p=\frac{n T}{3}$. For other forms of the constitutive relation for $\mathbf{J}_{\alpha}$, see e.g., $[30,10,15]$; they are all trivially related to each other.

The equation of motion obeyed by the velocity field can be written in the following standard form:

$$
\rho \frac{D V_{i}}{D t}=-\frac{\partial P_{i j}}{\partial x_{j}}-\rho \mathbf{g}
$$

where gravity is accounted for ( $\mathrm{g}$ being the gravitational acceleration), and $P_{i j}$ is the stress tensor, whose Navier-Stokes order assumes the form:

$$
P_{i j}=p \delta_{i j}-\mu D_{i j}-\eta_{B} \operatorname{div} \mathbf{V}
$$

where

$$
D_{i j}=\frac{1}{2}\left(\frac{\partial V_{i}}{\partial x_{j}}+\frac{\partial V_{j}}{\partial x_{i}}\right)-\frac{2}{3} \delta_{i j} \operatorname{div} \mathbf{V}
$$

is the symmetrized traceless rate of strain tensor, $\mu$, the shear viscosity, and $\eta_{B}$, the bulk viscosity (the latter vanishes in the dilute limit). Notice that $\mathrm{g}$ is formally a Euler order term (i.e. of the order of magnitude of a first order gradient); in other words the Navier-Stokes transport coefficients are independent of $\mathbf{g}[8]$.

The granular temperature field obeys the equation of motion:

$$
n \frac{D T}{D t}=T \operatorname{div} \mathbf{J}-\operatorname{div} \mathbf{Q}-2 P_{i j} \frac{\partial V_{i}}{\partial x_{j}}-\Gamma,
$$

where $\mathbf{J} \equiv \mathbf{J}_{A}+\mathbf{J}_{B}$ is the total particle flux, $\mathbf{Q}$ is the heat flux given by:

$$
\mathbf{Q}=\frac{5 \sqrt{6}}{18} \frac{1}{\sigma_{A B}^{2}} \frac{T^{3 / 2}}{\sqrt{m_{0}}}\left(\lambda^{T} \nabla \ln T+\lambda^{n} \nabla \ln n+\lambda^{c} \nabla \ln c\right)
$$


where $\sigma_{A B} \equiv \frac{\sigma_{A}+\sigma_{B}}{2}, m_{0} \equiv m_{A}+m_{B}$, and $\Gamma$ is the energy sink term, which accounts for the rate of loss of energy due to the inelasticity of the collisions (and therefore vanishes in the elastic limit). It is given by:

$$
\Gamma=\Gamma_{A}+\Gamma_{B}+\Gamma_{A B}
$$

where $\Gamma_{A}$ is the contribution of $A A$ collisions, $\Gamma_{B}$ is the contribution of $B B$ collisions, and $\Gamma_{A B}$ corresponds to $A B$ collisions; see explicit expressions for these entities below. Other forms of the same expression can be found in the above cited literature.

The preceding set of hydrodynamic equations, together with the expressions for the different fluxes and the source term, $\Gamma$, constitute a closed system of equations. It follows that a full hydrodynamic description is obtained by finding expressions for the transport coefficients $\left(\kappa_{\alpha}^{n}, \kappa_{\alpha}^{c}, \kappa_{\alpha}^{T}\right.$, $\lambda^{n}, \lambda^{c}, \lambda^{T}, \mu, \eta_{B}$, and of the sink term $\Gamma$ ), in terms of the fields and parameters of the problem. Note that [40] only $\Gamma$ has a nonvanishing contribution to zeroth order in the gradients of the fields (inelasticity acts in homogeneous systems as well) with corrections due to gradients, whereas the other fluxes vanish when the gradients do.

\subsection{Kinetic theoretical description}

Let $f_{\alpha}(\mathbf{v})$ denote the single particle distribution function (the spatial and temporal dependence of $f$ are suppressed for notational simplicity) of species $\alpha$. Following the standard definitions of kinetic theory, the number density for species $\alpha$ is given by:

$$
n_{\alpha}=\int f_{\alpha}(\mathbf{v}) d \mathbf{v}
$$

the corresponding mass density being $\rho_{\alpha}=m_{\alpha} n_{\alpha}$; recall that the overall number density is $n=$ $n_{A}+n_{B}$, and the overall mass density is $\rho \equiv \rho_{A}+\rho_{B}$. The velocity field of species $\alpha$ is given by:

$$
\mathbf{V}_{\alpha}=\frac{1}{n_{\alpha}} \int f_{\alpha}(\mathbf{v}) \mathbf{v} d \mathbf{v}
$$

We reiterate that $\mathbf{V}_{\alpha}$ is not a hydrodynamic field and it needs to be expressed as a functional of the hydrodynamic fields. The mixture's velocity field is:

$$
\mathbf{V}=\frac{1}{\rho}\left(\rho_{A} \mathbf{V}_{A}+\rho_{B} \mathbf{V}_{B}\right)
$$

The granular temperature of species $\alpha$ is defined by:

$$
T_{\alpha}=\frac{1}{n_{\alpha}} \int f_{\alpha}(\mathbf{v}) m_{\alpha}(\mathbf{v}-\mathbf{V})^{2} d \mathbf{v}
$$

Note that $T_{\alpha}$ is not a hydrodynamic field. Notice that the velocity fluctuations of each species are measured with respect to the (hydrodynamic) mixture's velocity field, not the species' velocity field. The mixture's granular temperature (average fluctuating kinetic energy multiplied by 2 ) is defined by:

$$
T \equiv \frac{1}{n}\left(n_{A} T_{A}+n_{B} T_{B}\right)
$$


The kinetic expression for the stress tensor (which excludes the collisional contribution; the latter comprises a finite density correction) is obtained by standard means, cf. e.g., [8]), the result being:

$$
P_{i j}=m_{A} \int f_{A}(\mathbf{v}) u_{i} u_{j} d \mathbf{v}+m_{B} \int f_{B}(\mathbf{v}) u_{i} u_{j} d \mathbf{v}
$$

where $\mathbf{u}_{i} \equiv \mathbf{v}_{i}-\mathbf{V}$ is the peculiar (fluctuating) velocity of particle $i$ (irrespective of its species). Similarly, the heat flux has two contributions, for obvious reasons:

$$
\mathbf{Q}=\int f_{A}(\mathbf{v}) m_{A} u^{2} \mathbf{u} d \mathbf{v}+\int f_{B}(\mathbf{v}) m_{B} u^{2} \mathbf{u} d \mathbf{v}
$$

The energy sink term is given by Eq. (2.11), the contributions to which are:

$$
\Gamma_{\alpha} \equiv \frac{\left(1-e_{\alpha \alpha}^{2}\right) m_{\alpha}}{8} \pi \sigma_{\alpha}^{2} \iint f_{\alpha}\left(\mathbf{v}_{1}\right) f_{\alpha}\left(\mathbf{v}_{2}\right)\left|v_{12}\right|^{3} d \mathbf{v}_{1} d \mathbf{v}_{2}
$$

and

$$
\Gamma_{A B} \equiv \varepsilon_{A B} \frac{m_{A B} \pi \sigma_{A B}^{2}}{2} \iint f_{A}\left(\mathbf{v}_{1}\right) f_{B}\left(\mathbf{v}_{2}\right)\left|\mathbf{v}_{12}\right|^{3} d \mathbf{v}_{1} d \mathbf{v}_{2} .
$$

where $m_{A B} \equiv \frac{m_{A} m_{B}}{m_{A}+m_{B}}$ is the reduced mass and $\sigma_{A B}=\frac{\sigma_{A}+\sigma_{B}}{2}$, as defined before. This completes the definitions of the entities one needs to calculate in order to obtain the constitutive relations.

\subsubsection{The Boltzmann Equation for a Granular Mixture}

As only dilute mixtures are considered here, the basic kinetic description is afforded by the Boltzmann equation. Actually, the description of a binary granular mixture requires two Boltzmann equations, one for each species. The derivation of these equations is a straightforward extension of that for the monodisperse case, and will not be presented here; see also [15].

The Boltzmann equations (for $\{\alpha, \beta\} \in\{A, B\}$ ) read:

$$
\mathcal{D} f_{\alpha} \equiv \frac{\partial f_{\alpha}}{\partial t}+\mathbf{v}_{1} \cdot \nabla f_{\alpha}=\mathcal{B}_{\alpha \alpha}\left(f_{\alpha}, f_{\alpha}, e_{\alpha \alpha}\right)+\mathcal{B}_{\alpha \beta}\left(f_{\alpha}, f_{\beta}, e_{\alpha \beta}\right)
$$

where $\alpha \neq \beta, f_{\alpha}(\mathbf{v})$ is the single distribution function for the particles of species $\alpha$, and the $\alpha-\beta$ species Boltzmann collision operator defined by:

$$
\mathcal{B}_{\alpha \beta}\left(f_{\alpha}, f_{\beta}, e_{\alpha \beta}\right)=\sigma_{\alpha \beta}^{2} \iint_{\mathbf{v}_{12} \cdot \mathbf{k}>0}\left[\frac{f_{\alpha}\left(\mathbf{v}_{1}^{\prime}\right) f_{\beta}\left(\mathbf{v}_{2}^{\prime}\right)}{e_{\alpha \beta}^{2}}-f_{\alpha}\left(\mathbf{v}_{1}\right) f_{\beta}\left(\mathbf{v}_{2}\right)\right]\left(\mathbf{v}_{12} \cdot \mathbf{k}\right) d \mathbf{v}_{2} d \mathbf{k}
$$

where $\sigma_{\alpha \beta}=\frac{\sigma_{\alpha}+\sigma_{\beta}}{2}$. Here $\mathbf{v}_{1}$ and $\mathbf{v}_{1}^{\prime}$ pertain to species $\alpha$, and $\mathbf{v}_{2}$ and $\mathbf{v}_{2}^{\prime}$ pertain to $\beta$. Notice that $\mathcal{B}_{\alpha \beta}\left(f_{\alpha}, f_{\beta}, e_{\alpha \beta}\right)$ depends on the coefficients of normal restitution both explicitly, as shown in Eq. (2.22), and implicitly through the velocity transformations between the precollisional and postcollisional velocities. 


\section{Computing the transport coefficients: the generating func- tion method}

\subsection{The Chapman-Enskog (CE) expansion}

The Chapman-Enskog expansion is a perturbative method for solving the Boltzmann equation. It consists of a gradient expansion of the distribution function around a reference state corresponding to a homogeneous solution of the Boltzmann equation (the Maxwellian equilibrium distribution function for elastic systems, or the distribution function corresponding to the Homogeneous Cooling State in the present case, see more below). One key assumption of the CE method is that the dependence of the distribution function on space and time is implicit through its functional dependence on the fields and that there is no additional space or time dependence (this amounts to assuming scale separation between the microscales and hydrodynamic scales, see also [17] concerning the range of validity of this assumption in the realm of granular gases). In the case at hand the pertinent hydrodynamic fields are $n_{A}, n_{B}, \mathbf{V}$, and $T$. This assumption allows one to expand the time derivative of the distribution function (left hand side of Eq. (2.21)) in powers of the gradients, by making use of the hydrodynamic equations, (2.3), (2.6), (2.9).

\subsubsection{The Homogeneous Cooling State (HCS)}

The homogeneous cooling state (HCS) of a binary granular gas mixture (much like the HCS for a monodisperse system) is defined as a state of vanishing velocity and homogeneous density fields, in which the distribution functions are rendered 'time independent' (recall that the energy decays because of inelasticity) by scaling the velocities by the thermal speed of the species comprising the mixture, thus defining a "scaling solution". This is also the 'asymptotic' state (in time) of a granular gas (ignoring instabilities). Since all gradients vanish in this state, the corresponding distribution function can be taken to serve as the zeroth order in the CE expansion. Using Eqs. (2.3), (2.6) and (2.9) one obtains for the HCS distributions, $f_{\alpha}^{H C S}$ :

$$
\frac{\partial f_{\alpha}^{(H C S)}}{\partial t}=\frac{\partial f_{\alpha}^{(H C S)}}{\partial T} \frac{\partial T}{\partial t}=-\frac{\Gamma}{n} \frac{\partial f_{\alpha}^{(H C S)}}{\partial T}
$$

where recall that $\Gamma=\Gamma_{A}+\Gamma_{B}+\Gamma_{A B}$, and $\Gamma_{\alpha}$ and $\Gamma_{A B}$ are given by Eqs. (2.19) and (2.20), respectively. The two coupled Boltzmann equations (2.21) reduce in this case to:

$$
\begin{aligned}
-\frac{\Gamma}{n} \frac{\partial f_{A}^{(H C S)}}{\partial T} & =\mathcal{B}_{A A}\left(f_{A}^{(H C S)}, f_{A}^{(H C S)}, e_{A A}\right)+\mathcal{B}_{A B}\left(f_{A}^{(H C S)}, f_{B}^{(H C S)}, e_{A B}\right) \\
-\frac{\Gamma}{n} \frac{\partial f_{B}^{(H C S)}}{\partial T} & =\mathcal{B}_{B B}\left(f_{B}^{(H C S)}, f_{B}^{(H C S)}, e_{B B}\right)+\mathcal{B}_{B A}\left(f_{B}^{(H C S)}, f_{A}^{(H C S)}, e_{A B}\right)
\end{aligned}
$$


where the Boltzmann operator $\mathcal{B}_{\alpha \beta}$ is defined in Eq. (2.22). These equations need to be solved subject to the 'constraints'

$$
\begin{aligned}
& \int f_{A} d \mathbf{u}=n_{A} \\
& \int f_{B} d \mathbf{u}=n_{B} \\
& \int f_{A} m_{A} u^{2} d \mathbf{u}+\int f_{B} m_{B} u^{2} d \mathbf{u}=n T
\end{aligned}
$$

the first two of which impose the respective number densities of the species and the last being an expression of the 'given' value of the temperature (the logic being that for 'given fields' at time, $t$, one finds their respective time derivatives).

\subsubsection{First order correction}

In order to compute the transport coefficients to Navier-Stokes order, we carry out the ChapmanEnskog expansion to first order in the gradients of the hydrodynamic fields, with the homogeneous cooling state distribution function serving as a zeroth order solution. The distribution function, $f_{\alpha}$, is thus written as: $f_{\alpha}=f_{\alpha}^{(H C S)}+f_{\alpha}^{K}$, where $f_{\alpha}^{K}$ is the first order perturbation in the gradient expansion, written for convenience as $f_{A}^{K}=f_{\alpha}^{M, \eta_{2}} \phi_{\alpha}^{K}$, where $f_{\alpha}^{M, \eta}=n_{\alpha}\left(\frac{\gamma_{\alpha}}{\pi}\right)^{\frac{3}{2}} e^{-\gamma_{\alpha} \eta u^{2}}$ is a modified Maxwellian, introduced for reasons explained below, $\gamma_{\alpha} \equiv \frac{3 m_{\alpha}}{2 T}$, and $\eta>0, \eta_{2}>0$ are constants (see more below). To first order in the gradients of the hydrodynamic fields, the two coupled Boltzmann equations for the corrections, $\phi_{\alpha}^{K}$, read:

$$
\begin{aligned}
& \mathcal{D}^{K} f_{A}=\left(L_{A A}^{(1)}+L_{A A}^{(2)}+L_{A B}^{(1)}\right) \phi_{A}^{K}+L_{A B}^{(2)} \phi_{B}^{K} \\
& \mathcal{D}^{K} f_{B}=L_{B A}^{(2)} \phi_{A}^{K}+\left(L_{B B}^{(1)}+L_{B B}^{(2)}+L_{B A}^{(1)}\right) \phi_{B}^{K}
\end{aligned}
$$

where

$$
\begin{aligned}
L_{\alpha \beta}^{(1)} \phi_{\alpha}^{K} & \equiv \mathcal{B}_{\alpha \beta}\left(f_{\alpha}^{M, \eta_{2}} \phi_{\alpha}^{K}, f_{\beta}^{(H C S)}, e_{\alpha \beta}\right) \\
L_{\alpha \beta}^{(2)} \phi_{\beta}^{K} & \equiv \mathcal{B}_{\alpha \beta}\left(f_{\alpha}^{(H C S)}, f_{\beta}^{M, \eta_{2}} \phi_{\beta}^{K}, e_{\alpha \beta}\right)
\end{aligned}
$$

are linearized Boltzmann operators, and $\mathcal{D}^{K} f_{\alpha}$ denotes the first order term in the expansion of $\mathcal{D} f_{\alpha}$, where recall that $\mathcal{D} \equiv \frac{\partial}{\partial t}+\mathbf{v} \cdot \nabla$. Following the CE approach:

$$
\begin{gathered}
\mathcal{D} f_{\alpha}=f_{\alpha}^{M, \eta}\left[\phi_{\alpha} \mathcal{D} \ln n_{\alpha}+2 \gamma_{\alpha} u_{i}\left(\eta \phi_{\alpha}-\phi_{\alpha}^{\prime}\right) \mathcal{D} V_{i}+\left(\gamma_{\alpha} u^{2}\left(\eta \phi_{\alpha}-\phi_{\alpha}^{\prime}\right)-\frac{3}{2} \phi_{\alpha}\right) \mathcal{D} \ln T\right. \\
\left.+c \frac{\partial \phi_{\alpha}}{\partial c} \mathcal{D} \ln c\right]+f_{\alpha}^{M, \eta_{2}} \mathcal{D} \Phi_{\alpha}^{K}+\Phi_{\alpha}^{K} f_{\alpha}^{M, \eta_{2}}\left[\mathcal{D} \ln n_{\alpha}+2 \gamma_{\alpha} u_{i} \eta_{2} \mathcal{D} V_{i}+\left(\gamma_{\alpha} u^{2} \eta_{2}-\frac{3}{2}\right) \mathcal{D} \ln T\right]
\end{gathered}
$$


Straightforward tensorial considerations can be used to determine the general form of the functions, $\phi_{\alpha}^{K}$ :

$$
\begin{aligned}
\phi_{\alpha}^{K} & =\Phi_{\alpha}^{K, T}\left(\gamma_{\alpha} u^{2}\right) \sqrt{\gamma_{\alpha}} \mathbf{u} \cdot \nabla \ln T+\Phi_{\alpha}^{K, n}\left(\gamma_{\alpha} u^{2}\right) \sqrt{\gamma_{\alpha}} \mathbf{u} \cdot \nabla \ln n+\Phi_{\alpha}^{K, c}\left(\gamma_{\alpha} u^{2}\right) \sqrt{\gamma_{\alpha}} \mathbf{u} \cdot \nabla \ln c \\
& +\Phi_{\alpha}^{K, V}\left(\gamma_{\alpha} u^{2}\right) \gamma_{\alpha}^{\frac{3}{2}} \overline{\mathbf{u u}}: \nabla \mathbf{V}+\Phi_{\alpha}^{0} \operatorname{div} \mathbf{V},
\end{aligned}
$$

where the functions $\Phi_{\alpha}^{K, T}, \Phi_{\alpha}^{K, c}, \Phi_{\alpha}^{K, n}, \Phi_{\alpha}^{K, V}$ and $\Phi_{\alpha}^{0}$ are isotropic functions of the rescaled peculiar velocity $\gamma_{\alpha} u^{2}$. It turns out that the $\Phi_{\alpha}^{0}$ vanishes; this becomes evident in the process of carrying out the CE expansion (since it has no 'source' on the right hand side of the pertinent equations). Note the definition of and notation for the traceless symmetric part of any second rank tensor, A: $\overline{A_{i j}} \equiv \frac{1}{2}\left(A_{i j}+A_{j i}-\frac{2}{3} \delta_{i j} A_{k k}\right) \cdot \mathcal{D}^{K} f_{\alpha}$ is given, using Eqs. (2.3), (2.6) and (2.9), by:

$$
\begin{aligned}
& \mathcal{D}^{K} f_{A}=\frac{\Gamma}{n T} f_{A}^{M, \eta_{2}}\left[\left(\gamma_{A} u^{2}\left(\Phi_{A}^{K, c}\right)^{\prime}-\left(\gamma_{A} u^{2} \eta_{2}-2\right) \Phi_{A}^{K, c}-c \frac{\partial \ln \widetilde{\Gamma}}{\partial c} \Phi_{A}^{K, T}\right) \sqrt{\gamma_{A}} u_{j} \frac{\partial \ln c}{\partial x_{j}}\right. \\
& +\left(\gamma_{A} u^{2}\left(\Phi_{A}^{K, n}\right)^{\prime}-\left(\gamma_{A} u^{2} \eta_{2}-2\right) \Phi_{A}^{K, n}-\Phi_{A}^{K, T}\right) \sqrt{\gamma_{A}} u_{j} \frac{\partial \ln n}{\partial x_{j}} \\
& +\left(\gamma_{A} u^{2}\left(\Phi_{A}^{K, T}\right)^{\prime}-\left(\gamma_{A} u^{2} \eta_{2}-2\right) \Phi_{A}^{K, T}-\frac{1}{2} \Phi_{A}^{K, T}\right) \sqrt{\gamma_{A}} u_{j} \frac{\partial \ln T}{\partial x_{j}} \\
& \left.+\left(\gamma_{A} u^{2}\left(\Phi_{A}^{K, V}\right)^{\prime}-\left(\gamma_{A} u^{2} \eta_{2}-3\right) \Phi_{A}^{K, V}\right) \gamma_{A}^{\frac{3}{2}} \overline{u_{i} u_{j}} \frac{\partial V_{i}}{\partial x_{j}}\right] \\
& +f_{A}^{M, \eta}\left[2 \gamma_{A}\left(\eta \phi_{A}-\phi_{A}^{\prime}\right) \overline{u_{i} u_{j}} \frac{\partial V_{i}}{\partial x_{j}}+\left(\phi_{A}-\frac{n m_{A}}{\rho}\left(\eta \phi_{A}-\phi_{A}^{\prime}\right)\right) u_{i} \frac{\partial \ln n}{\partial x_{i}}\right. \\
& +\left(\left(\gamma_{A} u^{2}-\frac{n m_{A}}{\rho}\right)\left(\eta \phi_{A}-\phi_{A}^{\prime}\right)-\frac{3}{2} \phi_{A}\right) u_{i} \frac{\partial \ln T}{\partial x_{i}} \\
& \left.+\left(\phi_{A}+c \frac{\partial \phi_{A}}{\partial c}\right) u_{j} \frac{\partial \ln c}{\partial x_{j}}\right] \\
& \mathcal{D}^{K} f_{B}=\frac{\Gamma}{n T} f_{B}^{M, \eta_{2}}\left[\left(\gamma_{B} u^{2}\left(\Phi_{B}^{K, c}\right)^{\prime}-\left(\gamma_{B} u^{2} \eta_{2}-2\right) \Phi_{B}^{K, c}-c \frac{\partial \ln \widetilde{\Gamma}}{\partial c} \Phi_{B}^{K, T}\right) \sqrt{\gamma_{B}} u_{j} \frac{\partial \ln c}{\partial x_{j}}\right. \\
& +\left(\gamma_{B} u^{2}\left(\Phi_{B}^{K, n}\right)^{\prime}-\left(\gamma_{B} u^{2} \eta_{2}-2\right) \Phi_{B}^{K, n}-\Phi_{B}^{K, T}\right) \sqrt{\gamma_{B}} u_{j} \frac{\partial \ln n}{\partial x_{j}} \\
& +\left(\gamma_{B} u^{2}\left(\Phi_{B}^{K, T}\right)^{\prime}-\left(\gamma_{B} u^{2} \eta_{2}-2\right) \Phi_{B}^{K, T}-\frac{1}{2} \Phi_{B}^{K, T}\right) \sqrt{\gamma_{B}} u_{j} \frac{\partial \ln T}{\partial x_{j}} \\
& \left.+\left(\gamma_{B} u^{2}\left(\Phi_{B}^{K, V}\right)^{\prime}-\left(\gamma_{B} u^{2} \eta_{2}-3\right) \Phi_{B}^{K, V}\right) \gamma_{B}^{\frac{3}{2}} \overline{u_{i} u_{j}} \frac{\partial V_{i}}{\partial x_{j}}\right]
\end{aligned}
$$




$$
\begin{aligned}
& +f_{B}^{M, \eta}\left[2 \gamma_{B}\left(\eta \phi_{B}-\phi_{B}^{\prime}\right) \overline{u_{i} u_{j}} \frac{\partial V_{i}}{\partial x_{j}}+\left(\phi_{B}-\frac{n m_{A}}{\rho}\left(\eta \phi_{B}-\phi_{B}^{\prime}\right)\right) u_{i} \frac{\partial \ln n}{\partial x_{i}}\right. \\
& +\left(\left(\gamma_{B} u^{2}-\frac{n m_{B}}{\rho}\right)\left(\eta \phi_{B}-\phi_{B}^{\prime}\right)-\frac{3}{2} \phi_{B}\right) u_{i} \frac{\partial \ln T}{\partial x_{i}} \\
& \left.+\left(-\frac{c}{1-c} \phi_{B}+c \frac{\partial \phi_{B}}{\partial c}\right) u_{j} \frac{\partial \ln c}{\partial x_{j}}\right]
\end{aligned}
$$

where primes denote derivatives with respect to the squared non dimensional peculiar velocity, $\gamma_{\alpha} u^{2}$. From Eqs. $(3.7,3.8)$ and Eqs. $(3.10,3.11)$, it follows that the functions $\Phi_{\alpha}^{K, c}, \Phi_{\alpha}^{K, n}, \Phi_{\alpha}^{K, T}$ and $\Phi_{\alpha}^{K, V}$ obey the following set of coupled equations:

$$
\begin{aligned}
& \left(L_{\alpha \alpha}^{(1)}+L_{\alpha \alpha}^{(2)}+L_{\alpha \beta}^{(1)}\right)\left\{\Phi_{\alpha}^{K, X}\left(\gamma_{\alpha} u^{2}\right) \sqrt{\gamma_{\alpha}} \mathbf{u}\right\}+L_{\alpha \beta}^{(2)}\left\{\Phi_{\beta}^{K, X}\left(\gamma_{\beta} u^{2}\right) \sqrt{\gamma_{\beta}} \mathbf{u}\right\} \\
& \quad-\frac{\sqrt{6 \pi}}{9} \frac{n \sigma_{\alpha \beta}^{2}}{\sqrt{m_{0}}} \widetilde{\Gamma} \sqrt{T} f_{\alpha}^{M, \eta_{2}}\left(\left(\mathcal{H}\left\{\Phi_{\alpha}^{K, X}\right\}+\xi^{X X}\right) \Phi_{\alpha}^{K, X}\left(\gamma_{\alpha} u^{2}\right) \sqrt{\gamma_{\alpha}} \mathbf{u}+\xi^{X T} \Phi_{\alpha}^{K, T}\left(\gamma_{\alpha} u^{2}\right) \sqrt{\gamma_{\alpha}} \mathbf{u}\right) \\
& =\mathbf{R}_{\alpha}^{X}
\end{aligned}
$$

for $X \in\{T, n, c\}$, and

$$
\begin{aligned}
\left(L_{\alpha \alpha}^{(1)}+L_{\alpha \alpha}^{(2)}+L_{\alpha \beta}^{(1)}\right) & \left\{\Phi_{\alpha}^{K, V}\left(\gamma_{\alpha} u^{2}\right) \gamma_{\alpha}^{\frac{3}{2}} \overline{\mathbf{u u}}\right\}+L_{\alpha \beta}^{(2)}\left\{\Phi_{\beta}^{K, V}\left(\gamma_{\beta} u^{2}\right) \gamma_{\beta}^{\frac{3}{2}} \overline{\mathbf{u u}}\right\} \\
& -\frac{\sqrt{6 \pi}}{9} \frac{n \sigma_{\alpha \beta}^{2}}{\sqrt{m_{0}}} \widetilde{\Gamma} \sqrt{T} f_{\alpha}^{M, \eta_{2}}\left(\left(\mathcal{H}\left\{\Phi_{\alpha}^{K, V}\right\}+3\right) \Phi_{\alpha}^{K, V}\left(\gamma_{\alpha} u^{2}\right) \gamma_{\alpha}^{\frac{3}{2}} \overline{\mathbf{u u}}\right)=\mathbf{R}_{\alpha}^{X}
\end{aligned}
$$

where the operator $\mathcal{H}$ is defined by

$$
\mathcal{H}\{\Phi\} \equiv \gamma_{\alpha} u^{2}\left((\ln \Phi)^{\prime}-\eta_{2}\right)
$$

and the coefficients $\xi^{X X}$ and $\xi^{X T}$ are given by: $\xi^{n n}=\xi^{c c}=2, \xi^{T T}=\frac{3}{4}, \xi^{c T}=-\frac{\partial \ln \tilde{\Gamma}}{\partial c}, \xi^{n T}=-1$. The tensors $\mathbf{R}_{\alpha}^{X}$ are given by:

$$
\begin{aligned}
\mathbf{R}_{A}^{c} & =f_{A}^{M, \eta}\left(\phi_{A}+c \frac{\partial \phi_{A}}{\partial c}\right) \mathbf{u} \\
\mathbf{R}_{B}^{c} & =f_{B}^{M, \eta}\left(-\frac{c}{1-c} \phi_{B}+c \frac{\partial \phi_{B}}{\partial c}\right) \mathbf{u} \\
\mathbf{R}_{\alpha}^{T} & =f_{\alpha}^{M, \eta}\left(\left(\gamma_{\alpha} u^{2}-\frac{n m_{\alpha}}{\rho}\right)\left(\eta \phi_{\alpha}-\phi_{\alpha}^{\prime}\right)-\frac{3}{2} \phi_{\alpha}\right) \mathbf{u} \\
\mathbf{R}_{\alpha}^{n} & =f_{\alpha}^{M, \eta}\left(\phi_{\alpha}-\frac{n m_{\alpha}}{\rho}\left(\eta \phi_{\alpha}-\phi_{\alpha}^{\prime}\right)\right) \mathbf{u} \\
\mathbf{R}_{\alpha}^{V} & =f_{\alpha}^{M, \eta} 2 \gamma_{\alpha}\left(\eta \phi_{\alpha}-\phi_{\alpha}^{\prime}\right) \overline{\mathbf{u} \mathbf{u}}
\end{aligned}
$$




\subsection{Sonine polynomial expansion and the generating function method}

In order to solve Eqs. $(3.2,3.3,3.12,3.13)$, the distribution functions $f_{A}^{(H C S)}, f_{A}^{K}, f_{B}^{(H C S)}$, and $f_{B}^{K}$ are approximated by truncated series in Sonine polynomials multiplying (modified, as explained below) Maxwellians (for $f_{A}^{(H C S)}$ and $f_{B}^{(H C S)}$ ), a similar expansion, in which the Maxwellians are multiplied by appropriate (contracted) tensors in the peculiar velocity being used for $f_{A}^{K}$, and $\left.f_{B}^{K}\right)$. For extreme values of the parameters, such as very low values of the coefficients of restitution or large mass ratios, the use of high order truncations of Sonine polynomial series is crucial, but forbiddingly tedious to carry out. To this end we employ a computer-aided method [32, 44] which exploits the fact that the Sonine polynomials can be derived from their respective generating functions, $G_{m}(x ; s) \equiv(1-s)^{-m-1} e^{-\frac{s}{1-s} x}=\sum_{p=0}^{\infty} s^{p} S_{m}^{p}(x)$ : Defining, for any set of variables $\left\{x_{1}, . ., x_{k}\right\}$ and integers $\left\{p_{1}, . ., p_{k}\right\}, \widetilde{\partial}_{x_{1}^{p_{1}}, . ., x_{k}^{p_{k}}}$ :

$$
\widetilde{\partial}_{x_{1}^{p_{1}}, . ., x_{k}^{p_{k}}} \equiv \lim _{x_{1}, . ., x_{k} \rightarrow 0} \frac{1}{p_{1} ! \ldots p_{k} !} \frac{\partial^{p_{1}+. .+p_{k}}}{\partial x_{1}^{p_{1}} . . \partial x_{k}^{p_{k}}}
$$

one has:

$$
S_{m}^{p}=\tilde{\partial}_{s^{p}} G_{m}(x ; s)
$$

A generating function for the action of a linear operator (such as the integrals required in the resolution the Chapman-Enskog expansion equations, see more details below) on a Sonine polynomial can therefore be easily calculated by evaluating its action on $G_{m}$. In addition, it turns out that most of the generating functions that are computed in the sequel can be obtained in terms of successive derivatives of a "super-generating" function $J_{\alpha \beta}^{(0)}$, see Appendix B, Eq. (B1). Increasing the order of the truncated series therefore amounts to computing higher order derivatives of known analytical functions, which can be achieved rather straightforwardly by a symbolic manipulator. The method is first applied below to the evaluation of the distribution function for the homogeneous cooling state, then to the computation of the transport coefficients.

The common way of finding approximate solutions of Eqs. (3.2,3.3) is to represent the functions, $f_{\alpha}$, by truncated Sonine polynomial series times Maxwellians in the respective (peculiar) velocities (often non-dimensionalization is invoked for sake of convenience). However, as numerically demonstrated in [5], this expansion does not converge, a feature that can be traced back [32] to the well known exponential tails of the HCS distribution function [11,34]. To overcome this difficulty, the following modified expansion [32] is employed:

$$
f_{\alpha}=n_{\alpha}\left(\frac{\gamma_{\alpha}}{\pi}\right)^{\frac{3}{2}} e^{-\eta \gamma_{\alpha} u^{2}} \phi_{\alpha}
$$

where $\phi_{\alpha} \equiv \sum_{p=0}^{\infty} h_{\alpha}^{p} S_{\frac{1}{2}}^{p}\left(\gamma_{\alpha} u^{2}\right)=\sum_{p=0}^{\infty} h_{\alpha}^{p} \tilde{\partial}_{s^{p}} G_{\frac{1}{2}}\left(\gamma_{\alpha} u^{2} ; s\right)$, and $\eta>0$ is a constant, i.e. $f_{\alpha}$ is represented by a truncated Sonine polynomial series times a Maxwellian in which the temperature is replaced by a "wrong" temperature $\frac{T}{\eta}$. This method produces convergent series when $\eta<0.5$ [32]. The standard (divergent, as it turns out) series, which corresponds to the choice $\eta=1$ ) is not useless, since (due to its asymptotic nature) it can be employed to obtain low order moments of the 
distribution functions, and it can also be employed for the derivation of constitutive relations for not-too-inelastic granular gases. It is however not suitable for the calculation of the distributions functions themselves. Upon substituting the form (3.16) in Eq. (3.2) and projecting on the Nth order Sonine polynomial $S_{\frac{1}{2}}^{N}\left(\gamma_{\alpha} u_{1}^{2}\right)=\widetilde{\partial}_{s^{N}} G\left(\gamma_{\alpha} u_{1}^{2}, s\right)$ one obtains:

$$
\begin{aligned}
\frac{\sqrt{\pi}}{3} \widetilde{\Gamma} \sum_{p=0}^{\infty} h_{A}^{p} \tilde{\partial}_{s^{p} w^{N}} \hat{R}(s, w) & =\frac{M_{A}^{3}}{\pi^{3}} \frac{\sigma_{A}^{2}}{\sigma_{A B}^{2}} \frac{n_{A}}{n} \sum_{p, q=0}^{\infty} \tilde{\partial}_{s^{p} t^{q} w^{N}} \hat{B}_{A A}(w, s, t) h_{A}^{p} h_{A}^{q} \\
& +\frac{M_{B}^{\frac{3}{2}} M_{A}^{\frac{3}{2}}}{\pi^{3}} \frac{n_{B}}{n} \sum_{p, q=0}^{\infty} \tilde{\partial}_{s^{p} t^{q} w^{N}} \hat{B}_{A B}(w, s, t) h_{A}^{p} h_{B}^{q} \\
\frac{\sqrt{\pi}}{3} \widetilde{\Gamma} \sum_{p=0}^{\infty} h_{B}^{p} \tilde{\partial}_{s^{p} w^{N}} \hat{R}(s, w) & =\frac{M_{B}^{3}}{\pi^{3}} \frac{\sigma_{B}^{2}}{\sigma_{A B}^{2}} \frac{n_{B}}{n} \sum_{p, q=0}^{\infty} \tilde{\partial}_{s^{p} t^{q} w^{N}} \hat{B}_{B B}(w, s, t) h_{B}^{p} h_{B}^{q} \\
& +\frac{M_{A}^{\frac{3}{2}} M_{B}^{\frac{3}{2}}}{\pi^{3}} \frac{n_{A}}{n} \sum_{p, q=0}^{\infty} \tilde{\partial}_{s^{p} t^{q} w^{N}} \hat{B}_{B A}(w, s, t) h_{B}^{p} h_{A}^{q}
\end{aligned}
$$

where $\tilde{\Gamma} \equiv \frac{9}{\sqrt{6 \pi}} \frac{\sqrt{m_{0}}}{n^{2} \sigma^{2}{ }^{3} T^{\frac{3}{2}}} \Gamma$. The generating function, $\hat{R}(s, w)$, which corresponds to the left hand side of Eqs. $(3.2,3.3)$ is:

$$
\begin{aligned}
\hat{R}(s, w) & \equiv \frac{1}{\pi^{\frac{3}{2}}} \int e^{-\eta u^{2}} G_{\frac{1}{2}}\left(u^{2}, w\right)\left[\frac{3}{2} G_{\frac{1}{2}}\left(u^{2}, s\right)-u^{2}\left(\eta G_{\frac{1}{2}}\left(u^{2}, s\right)-\frac{\partial}{\partial u^{2}} G_{\frac{1}{2}}\left(u^{2}, s\right)\right)\right] d \mathbf{u} \\
& =\frac{3}{2} \frac{(1-s) w}{(1-w s+(\eta-1)(1-w)(1-s))^{\frac{5}{2}}}
\end{aligned}
$$

and the generating function $\hat{B}_{\alpha \beta}(w, s, t)$, which corresponds to the Boltzmann operator, can be expressed in terms of the super-generating function defined in Eq. (B1):

$$
\begin{aligned}
\hat{B}_{\alpha \beta} & (w, s, t) \equiv \int G_{\frac{1}{2}}^{N}\left(M_{\alpha} u_{1}^{2}, w\right) \iint_{\mathbf{u}_{12} \cdot \mathbf{k}>0}\left[\frac{1}{e_{\alpha \beta}^{2}} e^{-\eta M_{\alpha} u_{1}^{\prime 2}-\eta M_{\beta} u_{2}^{\prime 2}} G_{\frac{1}{2}}\left(M_{\alpha} u_{1}^{\prime 2}, s\right) G_{\frac{1}{2}}\left(M_{\beta} u_{2}^{\prime 2}, t\right)\right. \\
- & \left.e^{-\eta M_{\alpha} u_{1}^{2}-\eta M_{\beta} u_{2}^{2}} G_{\frac{1}{2}}\left(M_{\alpha} u_{1}^{2}, s\right) G_{\frac{1}{2}}\left(M_{\beta} u_{2}^{2}, t\right)\right]\left(\mathbf{u}_{12} \cdot \mathbf{k}\right) d \mathbf{u}_{1} d \mathbf{u}_{2} d \mathbf{k} \\
= & \frac{1}{(1-s)^{\frac{3}{2}}(1-t)^{\frac{3}{2}}(1-w)^{\frac{3}{2}}} \\
& \times\left[\frac{1}{e_{\alpha \beta}^{2}} J_{\alpha \beta}^{(0)}\left(\frac{M_{\alpha} w}{1-w}, 0,\left(\frac{s}{1-s}+\eta\right) M_{\alpha},\left(\frac{t}{1-t}+\eta\right) M_{\beta}, 0,0,0\right)\right. \\
& \left.-J_{\alpha \beta}^{(0)}\left(M_{\alpha}\left(\eta+\frac{w}{1-w}+\frac{s}{1-s}\right),\left(\frac{t}{1-t}+\eta\right) M_{\beta}, 0,0,0\right)\right]
\end{aligned}
$$

The following relation between the non-dimensionalized sink term and the coefficients $\left\{h_{A}, h_{B}\right\}$ completes the system $(3.17,3.18)$, and is obtained by using the expression (3.16) in Eqs. (2.19) and 
(2.20):

$$
\begin{aligned}
\tilde{\Gamma} & =\varepsilon_{A A} \frac{n_{A}^{2}}{n^{2}} \frac{\sigma_{A}^{2}}{\sigma_{A B}^{2}} M_{A}^{4} \sum_{p, q=0}^{\infty} \tilde{\partial}_{s^{p} t^{q}} \hat{G}_{A A}^{\Gamma}(s, t) h_{A}^{p} h_{A}^{q}+4 \varepsilon_{A B} \frac{n_{A} n_{B}}{n^{2}} M_{A}^{\frac{5}{2}} M_{B}^{\frac{5}{2}} \sum_{p, q=0}^{\infty} \tilde{\partial}_{s^{p} t^{q}} \hat{G}_{A B}^{\Gamma}(s, t) h_{A}^{p} h_{B}^{q} \\
& +\varepsilon_{B B} \frac{n_{B}^{2}}{n^{2}} \frac{\sigma_{B}^{2}}{\sigma_{A B}^{2}} M_{B}^{4} \sum_{p, q=0}^{\infty} \tilde{\partial}_{s^{p} t^{q}} \hat{G}_{B B}^{\Gamma}(s, t) h_{B}^{p} h_{B}^{q}
\end{aligned}
$$

where the generating function $\hat{G}_{\alpha \beta}^{\Gamma}(s, t)$ is given by:

$$
\begin{aligned}
\hat{G}_{\alpha \beta}^{\Gamma}(s, t) & \equiv \iint e^{-\eta M_{\alpha} u_{1}^{2}} e^{-\eta M_{\beta} u_{2}^{2}} G_{\frac{1}{2}}\left(M_{\alpha} u_{1}^{2}, s\right) G_{\frac{1}{2}}\left(M_{\beta} u_{2}^{2}, t\right)\left|u_{12}\right|^{3} d \mathbf{u}_{1} d \mathbf{u}_{2} \\
& =\frac{\left(\frac{(1-t) M_{\alpha}}{(t+\eta(1-t))}+\frac{(1-s) M_{\beta}}{(s+\eta(1-s))}\right)^{\frac{3}{2}}}{(s+\eta(1-s))^{\frac{3}{2}}(t+(1-t) \eta)^{\frac{3}{2}} M_{\alpha}^{3} M_{\beta}^{3}}
\end{aligned}
$$

The system of equations $(3.17,3.18,3.22)$ for the coefficients $\left\{h_{A}, h_{B}, \tilde{\Gamma}\right\}$ is to be solved with the requirements (3.4), (3.5) and (3.6) that read, using (3.16):

$$
\begin{aligned}
& \sum_{p=0}^{\infty} \tilde{\partial}_{s^{p}}\left(\frac{h_{A}^{p}}{(s+(1-s) \eta)^{\frac{3}{2}}}\right)=1 \\
& \sum_{p=0}^{\infty} \tilde{\partial}_{s^{p}}\left(\frac{h_{B}^{p}}{(s+(1-s) \eta)^{\frac{3}{2}}}\right)=1 \\
& \sum_{p=0}^{\infty} \tilde{\partial}_{s^{p}}\left(\frac{(1-s)}{(s+(1-s) \eta)^{\frac{5}{2}}}\right)\left(\frac{n_{A}}{n} h_{A}^{p}+\frac{n_{B}}{n} h_{B}^{p}\right)=1
\end{aligned}
$$

A truncation of the above equations can be solved for any values of the parameters of the problem, by using standard solvers. Convergence is tested by checking that an increase in the order of truncation (we have checked up to the 25th order in the Sonine polynomial expansion in some cases) does not change the results (i.e., the desired coefficients) within a predetermined allowed error (1\% in most cases). As mentioned, when one does not need the distribution function but just small finite order moments thereof, one can employ the divergent expansion (i.e. use $\eta=1$ ). The above displayed algebraic equations for the HCS have been solved for a selection of values of the parameters. For near-elastic cases and $O(1)$ mass ratios the results for the low order moments converge (to within 1\%) for $N=4$ Sonine polynomials; the strongly inelastic cases and the cases in which the mass ratio is very large (or small) require up to 25 Sonine polynomials to obtain convergence. The moments converge even for $\eta=1$, whereas the distribution function only converges (as predicted by theory) for $\eta<\frac{1}{2}$ (in practice we used $\eta=0.4$ ). 
Eqs. (3.12) are solved using the same method as for the homogeneous cooling solution. The isotropic functions $\Phi_{\alpha}^{K, X}$ are expressed as truncated series ( at order $N$ ) of Sonine polynomials:

$$
\begin{aligned}
\Phi_{\alpha}^{K, T} & =\sum_{p=0}^{N} \frac{k_{\alpha}^{T,(p)}}{n \sigma_{A B}^{2}} S_{\frac{3}{2}}^{p}\left(\gamma_{\alpha} u^{2}\right)=\sum_{p=0}^{N} \frac{k_{\alpha}^{T,(p)}}{n \sigma_{A B}^{2}} \widetilde{\partial}_{t^{p}} G_{\frac{3}{2}}\left(t, \gamma_{\alpha} u^{2}\right) \\
\Phi_{\alpha}^{K, n} & =\sum_{p=0}^{N} \frac{k_{\alpha}^{n,(p)}}{n \sigma_{A B}^{2}} S_{\frac{3}{2}}^{p}\left(\gamma_{\alpha} u^{2}\right)=\sum_{p=0}^{N} \frac{k_{\alpha}^{n,(p)}}{n \sigma_{A B}^{2}} \widetilde{\partial}_{t^{p}} G_{\frac{3}{2}}\left(t, \gamma_{\alpha} u^{2}\right) \\
\Phi_{\alpha}^{K, c} & =\sum_{p=0}^{N} \frac{k_{\alpha}^{c,(p)}}{n \sigma_{A B}^{2}} S_{\frac{3}{2}}^{p}\left(\gamma_{\alpha} u^{2}\right)=\sum_{p=0}^{N} \frac{k_{\alpha}^{c,(p)}}{n \sigma_{A B}^{2}} \widetilde{\partial}_{t^{p}} G_{\frac{3}{2}}\left(t, \gamma_{\alpha} u^{2}\right) \\
\Phi_{\alpha}^{K, V} & =\sum_{p=0}^{N} \frac{k_{\alpha}^{V,(p)}}{n \sigma_{A B}^{2}} S_{\frac{5}{2}}^{p}\left(\gamma_{\alpha} u^{2}\right)=\sum_{p=0}^{N} \frac{k_{\alpha}^{V,(p)}}{n \sigma_{A B}^{2}} \widetilde{\partial}_{t^{p}} G_{\frac{5}{2}}\left(t, \gamma_{\alpha} u^{2}\right)
\end{aligned}
$$

which are substituted in Eqs. (3.12). The resulting set of equations is then projected on

$$
S_{\frac{3}{2}}^{N}\left(\gamma_{\alpha} u^{2}\right) \sqrt{\gamma_{\alpha}} \mathbf{u}=\tilde{\partial}_{w^{N}} G_{\frac{3}{2}}\left(w, \gamma_{\alpha} u^{2}\right) \sqrt{\gamma_{\alpha}} \mathbf{u},
$$

for $X \in\{T, c, n\}$ (i.e. multiplied by the functions, the result being integrated over the velocity), and

$$
S_{\frac{5}{2}}^{N}\left(\gamma_{\alpha} u^{2}\right) \gamma_{\alpha}^{3 / 2} \overline{\mathbf{u u}}=\tilde{\partial}_{w^{N}} G_{\frac{5}{2}}\left(w, \gamma_{\alpha} u^{2}\right) \gamma_{\alpha}^{3 / 2} \overline{\mathbf{u u}},
$$

for $X=V$, to yield the following linear system of equations for the coefficients $k_{\alpha}^{X,(p)}$ :

$$
\underline{\underline{\mathbf{M}}}^{K} \underline{\mathbf{k}}=\underline{\mathbf{R}}^{K}
$$

where $\underline{\mathbf{k}}$ is defined by: $\underline{\mathbf{k}} \equiv\left(k_{A}^{T,(0)}, . ., k_{A}^{T,(N)}, k_{B}^{T,(0)}, . ., k_{B}^{T,(N)}, k_{A}^{n,(0)}, . ., k_{A}^{n,(N)}, k_{B}^{n,(0)}, . ., k_{B}^{n,(N)}\right.$,

$$
\left.k_{A}^{c,(0)}, . ., k_{A}^{c,(N)}, k_{B}^{c,(0)}, . ., k_{B}^{c,(N)}, k_{A}^{V,(0)}, . ., k_{A}^{V,(N)}, k_{B}^{V,(0)}, . ., k_{B}^{V,(N)}\right)^{t}
$$

the superscript, $t$, standing for "transpose". The matrix elements, $M_{i j}^{K}$ and $R_{j}^{K}$ (and their generating functions), are given in Appendix A. The constraints, for $X \in\{T, n, c\}$ can be written as:

$$
\sum_{p=0}^{N} \widetilde{\partial}_{t^{p}} \frac{1}{\left(\eta_{2}(1-t)+t\right)^{\frac{5}{2}}} \times\left(\frac{n_{A}}{n} \sqrt{m_{A}} k_{A}^{X,(p)}+\frac{n_{B}}{n} \sqrt{m_{B}} k_{B}^{X,(p)}\right)=0
$$

\subsubsection{Constitutive Relations}

The constitutive relation are obtained by using the forms (3.9), (3.24-3.27), together with the solution $\left\{k_{\alpha}^{X,(q)}\right\}$, where $0 \leq q \leq N$, of the system (3.28), in the definitions of the diffusion velocity, 
heat flux and stress tensor. The resulting transport coefficients (like the coefficients $\left\{k_{\alpha}^{X,(q)}\right\}$ ), are non-trivial functions of the set of parameters

$$
\mathcal{S} \equiv\left\{\left\{\varepsilon_{\alpha \beta}\right\}, M_{A} \equiv \frac{m_{A}}{m_{A}+m_{B}}, \frac{\sigma_{A}}{\sigma_{A}+\sigma_{B}}, c\right\} .
$$

The pressure $p$ is given by:

$$
p=\frac{n T}{3},
$$

the bulk viscosity vanishes, $\eta_{B}=0$, and the shear viscosity is given by:

$$
\mu=-\frac{\sqrt{6}}{24} \frac{\sqrt{T m_{0}}}{\sigma_{A B}^{2}} \sum_{p=0}^{N} \widetilde{\partial}_{s^{p}} \frac{1}{\left(\eta_{2}(1-s)+s\right)^{\frac{7}{2}}}\left(\frac{n_{A}}{n} \sqrt{M_{A}} k_{A}^{V,(p)}+\frac{n_{B}}{n} \sqrt{M_{B}} k_{B}^{V,(p)}\right)
$$

The transport coefficients for the diffusion flux are given by:

$$
\kappa_{A}^{X}=\sum_{p=0}^{N} \widetilde{\partial}_{t^{p}} \frac{k_{A}^{X,(p)}}{\left(\eta_{2}(1-t)+t\right)^{\frac{5}{2}}}
$$

and the transport coefficients for the heat flux are:

$$
\lambda^{X}=\sum_{p=0}^{N} \widetilde{\partial}_{t^{p}} \frac{(1-t)}{\left(\eta_{2}(1-t)+t\right)^{\frac{7}{2}}}\left(\frac{n_{A}}{n \sqrt{M_{A}}} k_{A}^{X,(p)}+\frac{n_{B}}{n \sqrt{M_{B}}} k_{B}^{X,(p)}\right)
$$

The need for high order Sonine polynomial expansions to obtain accurate transport coefficients is illustrated in Fig. (1), in which results for the coefficient $\kappa_{A}^{n}$ (normalized by its elastic value) as a function of the degree of inelasticity are depicted (here, $\sigma_{A}=\sigma_{B}, n_{A}=n_{B}$, and all coefficients of restitution are taken to be equal: $\varepsilon \equiv \varepsilon_{A A}=\varepsilon_{A B}=\varepsilon_{B B}$ ). The most accurate results presented in this figure are obtained from a seventh order truncation of the Sonine polynomial expansions, cf. Eqs. (3.24)-(3.27), and depicted by the fat dots in the figure. These results compare well with those obtained from a sixth order truncation (circles), except for degrees of inelasticity larger than 0.7 , where even higher orders in the expansion are needed. They are different from the results obtained in ref. [15], which are referred to as the "standard" expansion and those of the modified expansion, [16]. Note that the "standard" and modified expansions agree with each other for low values of the coefficient of restitution and they both employ the leading order in the Sonine polynomial approximation. An additional interesting observation can be made on the basis of the expansion in powers of $\varepsilon$, referred to in the figure as the $\varepsilon$-expansion. The leading (linear in $\varepsilon$ ) order in this expansion should agree with the correct results for low values of $\varepsilon$, and, in particular with the slope at the origin of the graphs in Fig. (1). It turns out that to obtain a converged result of the linear order in the $\varepsilon$-expansion it is sufficient to truncate the corresponding Sonine polynomial expansion at second order. Indeed this result (depicted by the symbol $\mathrm{x}$ ) agrees with the exact result near the origin and surprisingly also much beyond it. On the other hand the use of the lowest order in the Sonine expansion alone in the $\varepsilon$-expansion (dashes) does not agree with the accurate result 
but it does agree with the other results obtained by a truncation at the lowest order in the Sonine polynomial expansion. It follows that, as mentioned, the order of truncation does matter.

Through formally valid for all values of the parameters characterizing the particles and their collisions (masses, diameters and coefficients of normal restitution), the validity of above transport coefficients (or more generally of the constitutive relations $(2.5,2.7,2.10)$ ) may be limited due to the underlying assumptions of the CE expansion, in particular that of time scale separation (expressed through the fact that the distribution functions depend on time through the hydrodynamic fields only). For strongly inelastic systems, or extreme values of the mass and diameter ratios, scale separation can be violated, invalidating the above results for the transport coefficients and possibly the entire description to Navier-Stokes order [41].

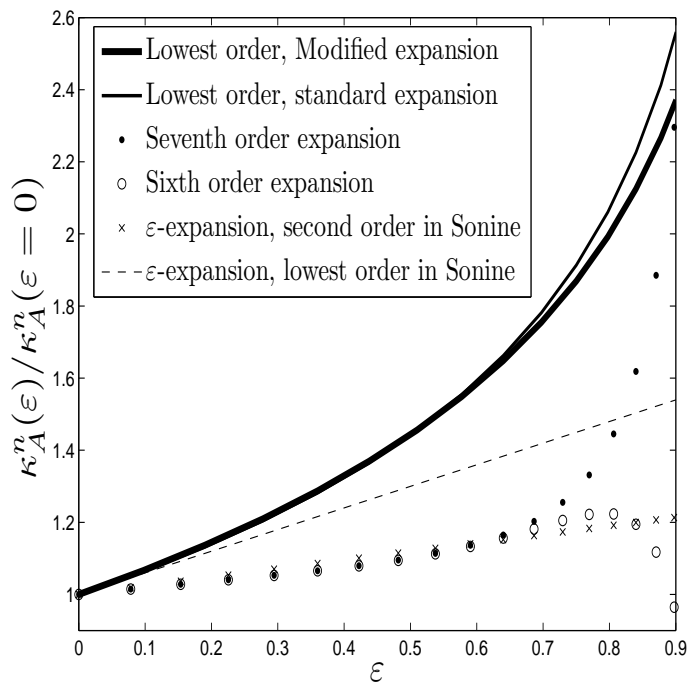

Figure 1: Comparison between various predictions for the coefficient $\kappa_{A}^{n}$ (normalized by its elastic value), plotted as a function of $\varepsilon \equiv \varepsilon_{A A}=\varepsilon_{A B}=\varepsilon_{B B}$, for $\sigma_{A}=\sigma_{B}, n_{A}=n_{B}$, and a mass ratio, $m_{A} / m_{B}=2$. The thick solid line corresponds to the "modified Sonine expansion" [16] and the thin solid line depicts the results of the "standard" Sonine expansion [15], both to lowest order in the Sonine polynomial expansion. Dots and circles correspond to the 7th and 6th order truncations of the Sonine polynomials expansion, respectively. The dashed line corresponds to the lowest order in the Sonine polynomial expansion and linear order in the $\varepsilon$ expansion, and the $\mathrm{x}$-symbols correspond to the second order in the Sonine polynomial expansion and linear order in $\varepsilon$, see [43]. The predictions corresponding to low and high order truncations differ significantly from each other. Note that convergence is not attained at high inelasticity even at the seventh order truncation in the Sonine polynomial expansion. More comments can be found in the text. 


\section{Inelasticity-induced segregation in vibrated binary granular mixtures}

This section is devoted to a study of the direct influence of inelasticity on the properties of binary granular gas mixtures, namely the fact that the species may segregate even when they differ only in their respective coefficients of restitution. A similar result has been predicted in [43] and observed in molecular dynamic simulations in [6]. Here we employ our computer-aided method to extend the theory of [43] to all values of the coefficients of restitution. Before considering the problem of a vibrated vertically vibrated mixtures of particles, we first focus on the influence of the inelasticity on the mechanisms of segregation in this class of systems. Consider a non-convecting $(\mathbf{V}=0)$ steady state of a mixture. Clearly, the diffusion flux vanishes in this state. In this case, using Eq. (3.29), the equation for the momentum density (Eq. (2.6)) reduces to:

$$
\nabla p=-\rho \mathbf{g}
$$

which can be rewritten, since $p=\frac{n T}{3}$, and $\rho=\left(m_{A} c+m_{B}(1-c)\right) n$, as:

$$
\nabla \ln n=-\nabla \ln T-\frac{3}{T}\left(\left(m_{A}-m_{B}\right) c+m_{B}\right) \mathbf{g} .
$$

Upon substituting Eq. (4.2) in Eq. (2.5), and using the fact that in a non-convecting state the diffusive fluxes vanish, i.e. $\mathbf{J}_{A}=0$, one obtains the following relation between the temperature and concentration gradients:

$$
\left.\nabla \ln c=\frac{\kappa_{A}^{n}-\kappa_{A}^{T}}{\kappa_{A}^{c}} \nabla \ln T+\frac{3}{T} \frac{\kappa_{A}^{n}}{\kappa_{A}^{c}}\left(\left(m_{A}-m_{B}\right) c+m_{B}\right)\right) \mathbf{g}
$$

Define the rescaled length: $\xi=\int_{0}^{z} \frac{\left(m_{A}+m_{B}\right) g}{T\left(z^{\prime}\right)} d z^{\prime}$. When the temperature and concentration gradients vary only in the $z$ direction (defined by $-\mathbf{g}$ ) one obtains from Eq. (4.3):

$$
\frac{\partial \ln c}{\partial \xi}=\alpha_{A B} \frac{\partial \ln T}{\partial \xi}+\beta_{A B}
$$

where

$$
\alpha_{A B} \equiv \frac{\kappa_{A}^{n}-\kappa_{A}^{T}}{\kappa_{A}^{c}}
$$

and

$$
\beta_{A B}=3 \frac{\kappa_{A}^{n}}{\kappa_{A}^{c}}\left(\left(M_{A}-M_{B}\right) c+M_{B}\right)
$$

Eq. (4.4) indicates that segregation in this case is driven both by the temperature gradient and gravity. The value of the coefficient $\alpha_{A B}$ determines the degree of segregation induced by the temperature gradient, while its sign determines the direction of segregation: when $\alpha_{A B}>0$ the $A$ particles tends to concentrate in the hotter regions. Similarly, the effect of gravity is described by the coefficient $\beta_{A B}$ : its value indicates the degree of segregation due to the influence of gravity, 

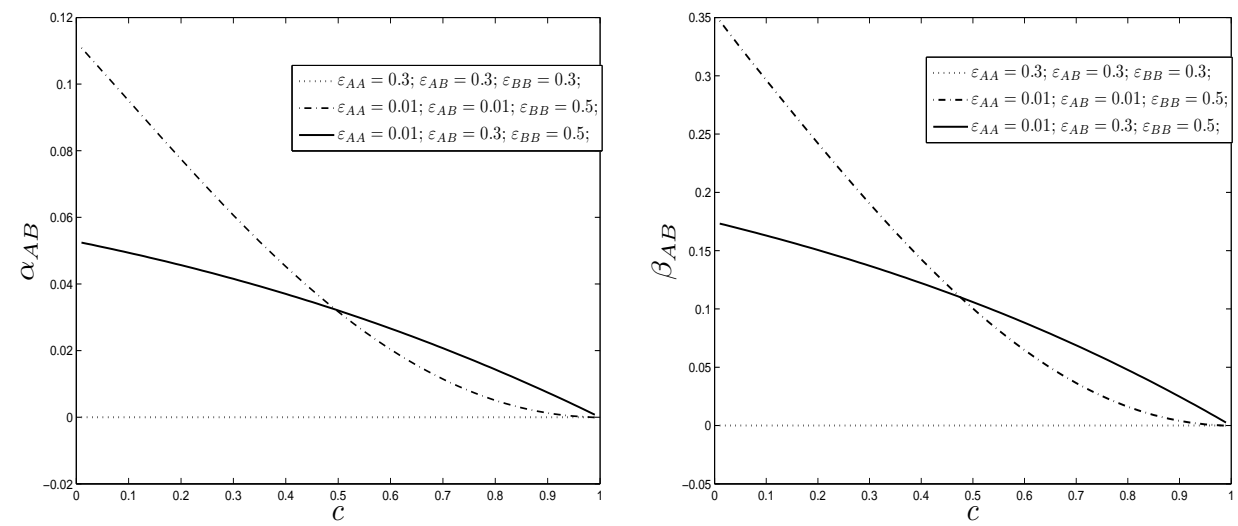

Figure 2: LEFT: Plots of the thermal coefficient, $\alpha_{A B}$, see Eq. (4.5), as a function of the concentration, $c \equiv \frac{n_{A}}{n}$, for different values of the degrees of inelasticity, as detailed in the inset. Here $m_{A}=m_{B}$ and $\sigma_{A}=\sigma_{B}$. Note that $\alpha_{A B} \neq 0$ when the coefficients of restitution are different. As expected $\alpha_{A B}=0$ when the coefficients of restitution are all equal, since the system is then monodisperse. The positive sign of $\alpha_{A B}$ indicates that the A-particles (whose collisions are more elastic in this case) move up the temperature gradient and therefore the more inelastically colliding (or B, in this case) particles tends to concentrate in the 'colder' regions. RIGHT: Plots of the thermal coefficient, $\beta_{A B}$, see Eq. (4.6), as a function of the concentration, $c \equiv \frac{n_{A}}{n}$, for the same parameters as in LEFT. The sign of $\beta_{A B}$ indicates that gravity acts to move the more inelastically colliding particles downward.

while its sign determines the direction of the segregation: when $\beta_{A B}>0$ the particles $A$ tends to concentrate near the top of the system. Notice that the influence of gravity described by $\beta_{A B}>0$ is different from buoyancy in the dilute limit considered here. In particular, it has been shown [42] that the sign of $\beta_{A B}$ possesses a non trivial dependence on the sizes of the particles, which constitutes a qualitative difference with respect to the elastic (dilute) limit where gravity always acts to move the lighter particles upward. In contrast, the inelasticity of the collisions introduces an additional dependence of $\beta_{A B}$ on the sizes of the particles that acts to move the smaller (not necessarily lighter) grains upward. Here we consider the case $m_{A}=m_{B}$ and $\sigma_{A}=\sigma_{B}$. Fig. (2) presents plots of the coefficients $\alpha_{A B}$ (LEFT) and $\beta_{A B}$ (RIGHT) as functions of the concentration, $c$, of the $A$ particles, for different values of the degrees of inelasticity. When the degrees of inelasticity are identical both coefficients vanish as expected, since the system is then monodisperse. In contrast, when the coefficients of normal restitution are different form each other, temperature gradients and gravity induce concentration gradients. Their influence increases with inelasticity and is enhanced by the difference in the elastic properties of the particles. The values of $\alpha_{A B}$ presented in Fig. (2, LEFT) comprise an extension to strong inelasticity of the predictions of [43], where the analysis is limited to slightly inelastic systems. While quantitative differences are observed, the qualitative features are similar: the sign of $\alpha_{A B}$ shows that the more inelastic particles tend to concentrate in the hotter regions. The sign of $\beta_{A B}$ (Fig. (2, RIGHT)) indicates that gravity drives 
the more inelastic particles downward. This effect is consistent with the behavior observed in MD simulations [6].

Consider next the consequences of the inelasticity driven segregation mechanism for a vertically vibrated binary mixture of particles differing only in their elastic properties (and subject to gravity), where energy is supplied at the floor or bottom of the system. As mentioned, such systems possess a minimum of temperature as a function of height. The induced temperature gradient tends to drive the more inelastic particles toward the region where the temperature is minimal, therefore competing with the influence of gravity. The result is a sandwich-like segregated configuration, which bears similarities to the mass segregation arrangements observed in vibrated systems of particles of similar size and different masses [43, 42]. In the simple case when there is no convection and the system is infinite and homogeneous in horizontal planes, the equations of motion reduce to:

$$
\begin{gathered}
\mathbf{J}_{A}=0 \\
\frac{d p}{d z}=-\rho g
\end{gathered}
$$

and, cf. Eq. (2.9):

$$
-\frac{\partial Q_{z}}{\partial z}=\Gamma
$$

Measuring height in terms of the rescaled local pressure $\widehat{p}=\frac{\sigma_{A B}^{2}}{m_{0} g} p$ (this is allowed since the pressure is monotonically decreasing with height), and defining $\hat{\eta} \equiv-\widehat{p} \frac{\partial \ln \sqrt{T}}{\partial \widehat{p}}$, Eqs. (4.7-4.9) reduce to the following two first order coupled ODE's [43]:

$$
\begin{aligned}
& \frac{\partial \hat{\eta}}{\partial \widehat{p}}=F_{1} \frac{\hat{\eta}^{2}}{\widehat{p}}-F_{2} \frac{\hat{\eta}}{\widehat{p}}+\frac{F_{3}}{\widehat{p}}-F_{4} \widehat{p} \\
& \frac{\partial c}{\partial \widehat{p}}=-F_{5} \frac{\hat{\eta}}{\widehat{p}}-\frac{F_{6}}{\widehat{p}}
\end{aligned}
$$

where $F_{1} \equiv 1+\frac{F_{7}^{\prime} F_{5}}{F_{7}}, F_{2} \equiv-\frac{F_{7}^{\prime} F_{6}}{F_{7}}-\frac{F_{8}^{\prime} F_{5}}{F_{7}}-\frac{F_{8}}{F_{7}}, F_{3} \equiv \frac{F_{8}^{\prime} F_{6}}{F_{7}}$ and $F_{4} \equiv-\frac{6 \sqrt{\pi}}{5} \frac{\tilde{\Gamma}}{F_{7}\left(\left(M_{A}-M_{B}\right) c+M_{B}\right)}$, $F_{5} \equiv 2\left(\frac{\kappa_{A}^{n}-\kappa_{A}^{T}}{\kappa_{A}^{c}}\right) c, F_{6} \equiv \frac{\kappa_{A}^{n}}{\kappa_{A}^{c}} c, F_{7} \equiv 6\left(\lambda^{T}-\lambda^{n}+\lambda^{c} \alpha_{A B}\right)\left(M_{A} c+M_{B}(1-c)\right)$, $F_{8} \equiv 3\left(\lambda^{c} \frac{\kappa_{A}^{n}}{\kappa_{A}^{c}}-\lambda^{n}\right)\left(M_{A} c+M_{B}(1-c)\right)$, and $F_{i}^{\prime} \equiv \frac{\partial F_{i}}{\partial c}$, where recall that $\Gamma=\frac{\sqrt{6 \pi}}{9} \frac{n^{2} \sigma_{A B}^{2}}{\sqrt{m_{0}}} T^{\frac{3}{2}} \tilde{\Gamma}$, $m_{0} \equiv m_{A}+m_{B}, M_{B}=1-M_{A}$ (recall that $\left.M_{A} \equiv \frac{m_{A}}{m_{A}+m_{B}}\right)$. All $\left\{F_{i}\right\}$ are functions of the set of dimensionless parameters, $\mathcal{S} \equiv\left\{\left\{\varepsilon_{\alpha \beta}\right\}, M_{A} \equiv \frac{m_{A}}{m_{A}+m_{B}}, \frac{\sigma_{A}}{\sigma_{A}+\sigma_{B}}, c\right\}$. Eqs. (4.10, 4.11) can be readily numerically solved, for given values of $c$ and $\hat{\eta}$ at a given value of $\hat{p}$ as "initial conditions" (or equivalently, given the average value of $c$ and the heat flux at the floor). Fig. (3) presents a solution for the case $m_{A}=m_{B}, \sigma_{A}=\sigma_{B}, \varepsilon_{A A}=0.01, \varepsilon_{A B}=0.3$, and $\varepsilon_{B B}=0.5$. The concentration, $c$, of the $A$ particles, is plotted as a function of the rescaled pressure $\hat{p}$ (see Fig. (3, RIGHT). The profile shows a minimum of the concentration of the $A$ (more elastic) particles at a finite pressure, i.e. the more inelastic $(B)$ particles concentrate in a layer trapped between two layers of more elastic ones, in a sandwich-like arrangement. In addition, the maximal value of the density of the more inelastic particles is situated below (at higher pressure, see Fig. 

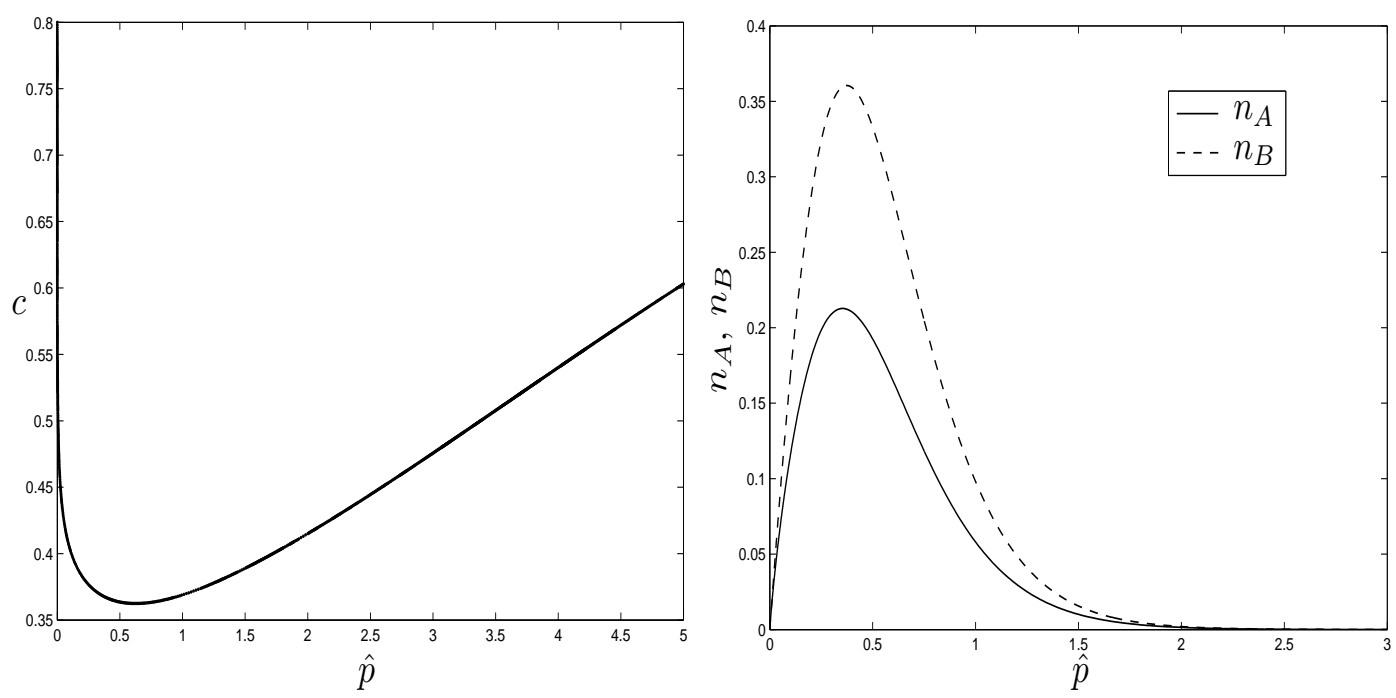

Figure 3: LEFT: Plot of the concentration, $c$, of the $A$ particles, as a function of the rescaled pressure $\hat{p}$. Here $m_{A}=m_{B}, \sigma_{A}=\sigma_{B}, \varepsilon_{A A}=0.01, \varepsilon_{A B}=0.3$, and $\varepsilon_{B B}=0.5$, i.e. the particles differ only in their respective coefficients of restitution. The concentration profile shows that the system arranges in a sandwich-like configuration, the more inelastic particles concentrating in between two layers of more elastic particles. RIGHT: The number densities, $n_{A}$, and $n_{B}$, as a function of $\hat{p}$ for the same system.

(3, RIGHT) ) that of the more elastic particles, in qualitative agreement with the the molecular dynamic simulations of [6]. Note that the maximal pressure depends on the total weight of the particles in the system and it occurs, of course, at the floor. This determines a range of pressures (starting at zero at infinite height above the floor) for a given systems. When this range includes the pressure corresponding to the minimal value of the concentration, $c$, one obtains a sandwich configuration, else one doesn't.

\section{Concluding remarks}

We have shown how a computer aided method can be exploited for the purpose of carrying out the tedious calculations involved in the performance of a Chapman-Enskog expansion for a dilute binary granular gas mixture. The method allows one to obtain numerically converged results for practically any set of values of the pertinent parameters. We presented an application to a binary system in which the only difference between the granular species is their collisional properties. This was shown to suffice to produce segregation in general and a sandwich-like pattern in the case of a vertically vibrated system. Another, quite surprising result is that even a classical effect such as buoyancy is affected by inelasticity. 


\section{Acknowledgments}

This work was partially supported by grant no. 412/08 from the Israel Science Foundation (ISF), grant no. 2004/391 from the US-Israel Binational Science Foundation (BSF) and grant no. I-795166.10/2003 from the German - Israeli Foundation for Scientific Research and Development (GIF). We are grateful to V. Garzó for providing us with some of the data presented in Fig. (1).

\section{A Matrix elements}

This appendix provides the matrix elements needed to solve the linear system, Eq. (3.28), corresponding to a truncation of the Sonine polynomial expansion at order N. The matrix elements $M_{i, j}^{K}$ for $1 \leq i \leq 6 N+6,1 \leq j \leq 6 N+6$ have been calculated by substituting the expressions (3.24-3.26) into the left hand side of Eq. (3.12), multiplying by the functions

$$
S_{\frac{3}{2}}^{i}\left(\gamma_{\alpha} u^{2}\right) \sqrt{\gamma_{\alpha}} \mathbf{u}=\tilde{\partial}_{w^{i}} G_{\frac{3}{2}}\left(w, \gamma_{\alpha} u^{2}\right) \sqrt{\gamma_{\alpha}} \mathbf{u}
$$

and integrating over the velocities. The matrix elements $M_{i, j}^{K}$ for $6 N+7 \leq i \leq 8 N+8$, $6 N+7 \leq j \leq 8 N+8$ have been calculated by substituting the expressions (3.27) into the left hand side of Eq. (3.13), multiplying by the functions, $S_{\frac{5}{2}}^{i}\left(\gamma_{\alpha} u^{2}\right) \gamma_{\alpha}^{3 / 2} \overline{\mathbf{u u}}=\tilde{\partial}_{w^{i}} G_{\frac{5}{2}}\left(w, \gamma_{\alpha} u^{2}\right) \gamma_{\alpha}^{3 / 2} \overline{\mathbf{u u}}$, and integrating over the velocities. The results are expressed below in terms of derivatives of the generating functions, $\hat{L}_{\alpha \beta}^{(i, j)}(w, s, r)$, given in Eqs. (A4-A7), and the generating functions, $\hat{H}^{V}(w, t)$, $\hat{H}(w, t)$, and $\hat{Z}(w, t)$, given in Eqs. (A1-A3). For $1 \leq i \leq N+1,1 \leq j \leq N+1$, the non zero elements of the $(8 N+8) \times(8 N+8)$ matrix $\underline{\underline{M}}^{K}$ are:

$$
\begin{aligned}
M_{i, j}^{K}= & \left(\frac{n_{A}}{n} \frac{\sigma_{A}^{2}}{\sigma_{A B}^{2}} M_{A}^{4} \sum_{p} h_{A}^{p} \tilde{\partial}_{w^{i}, s^{p}, r^{j}}\left(\hat{L}_{A A}^{(1,1)}(w, s, r)+\hat{L}_{A A}^{(1,2)}(w, s, r)\right)\right. \\
& +\frac{n_{B}}{n} M_{A}^{\frac{5}{2}} M_{B}^{\frac{3}{2}} \sum_{p} h_{B}^{p} \tilde{\partial}_{w^{i}, s^{p}, r^{j}} \hat{L}_{A B}^{(1,1)}(w, s, r) \\
& \left.-\frac{\pi^{\frac{7}{2}}}{3} \widetilde{\Gamma}\left(\tilde{\partial}_{w^{i} t^{j}} \hat{H}(w, t)+\frac{3}{2} \tilde{\partial}_{w^{i} t^{j}} \hat{Z}(w, t)\right)\right) \\
& M_{i, j+(N+1)}^{K}=\frac{n_{B}}{n} M_{A}^{2} M_{B}^{2} \sum_{p} h_{A}^{p} \tilde{\partial}_{w^{i}, s^{p}, r^{j}} \hat{L}_{A B}^{(1,2)}(w, s, r) \\
& M_{i+(N+1), j}^{K}=\frac{n_{A}}{n} M_{B}^{2} M_{A}^{2} \sum_{p} h_{B}^{p} \tilde{\partial}_{w^{i}, s^{p}, r^{j}} \hat{L}_{B A}^{(1,2)}(w, s, r)
\end{aligned}
$$




$$
\begin{aligned}
& M_{i+(N+1), j+(N+1)}^{K}=\frac{n_{B}}{n} \frac{\sigma_{B}^{2}}{\sigma_{A B}^{2}} M_{B}^{4} \sum_{p} h_{B}^{p} \tilde{\partial}_{w^{i}, s^{p}, r^{j}}\left(\hat{L}_{B B}^{(1,1)}(w, s, r)+\hat{L}_{B B}^{(1,2)}(w, s, r)\right) \\
& +\frac{n_{A}}{n} M_{B}^{\frac{5}{2}} M_{A}^{\frac{3}{2}} \sum_{p} h_{A}^{p} \tilde{\partial}_{w^{i}, s^{p}, r^{j}} \hat{L}_{B A}^{(1,1)}(w, s, r) \\
& -\frac{\pi^{\frac{7}{2}}}{3} \widetilde{\Gamma}\left(\tilde{\partial}_{w^{i} t^{j}} \hat{H}(w, t)+\frac{3}{2} \tilde{\partial}_{w^{i}, t^{j}} \hat{Z}(w, t)\right) \\
& M_{i+(2 N+2), j}^{K}=\frac{\pi^{\frac{7}{2}}}{3} \widetilde{\Gamma} \sum_{q} \tilde{\partial}_{w^{i}, t^{j}} \hat{Z}(w, t) \\
& M_{i+(2 N+2), j+(2 N+2)}^{K}=\left(\frac{n_{A}}{n} \frac{\sigma_{A}^{2}}{\sigma_{A B}^{2}} M_{A}^{4} \sum_{p} h_{A}^{p} \tilde{\partial}_{w^{i}, s^{p}, r^{j}}\left(\hat{L}_{A A}^{(1,1)}(w, s, r)+\hat{L}_{A A}^{(1,2)}(w, s, r)\right)\right. \\
& +\frac{n_{B}}{n} M_{A}^{\frac{5}{2}} M_{B}^{\frac{3}{2}} \sum_{p} h_{B}^{p} \tilde{\partial}_{w^{i}, s^{p}, r^{j}} \hat{L}_{A B}^{(1,1)}(w, s, r) \\
& \left.-\frac{\pi^{\frac{7}{2}}}{3} \widetilde{\Gamma}\left(\tilde{\partial}_{w^{i}, t^{j}} \hat{H}(w, t)+2 \tilde{\partial}_{w^{i} t^{j}} \hat{Z}(w, t)\right)\right) \\
& M_{i+(2 N+2), j+(3 N+3)}^{K}=\frac{n_{B}}{n} M_{A}^{2} M_{B}^{2} \sum_{p} h_{A}^{p} \tilde{\partial}_{w^{i}, s^{p}, r^{j}} L_{A B}^{(1,2)}(w, s, r) \\
& M_{i+(3 N+3), j+(N+1)}^{K}=\frac{\pi^{\frac{7}{2}}}{3} \widetilde{\Gamma} \sum_{q} \tilde{\partial}_{w^{i} t^{j}} \hat{Z}(w, t) \\
& M_{i+(3 N+3), j+(2 N+2)}^{K}=\frac{n_{A}}{n} M_{B}^{2} M_{A}^{2} \sum_{p} h_{B}^{p} \tilde{\partial}_{w^{i}, s^{p}, r^{j}} \hat{L}_{B A}^{(1,2)}(w, s, r) \\
& M_{i+(3 N+3), j+(3 N+3)}^{K}=\frac{n_{B}}{n} \frac{\sigma_{B}^{2}}{\sigma_{A B}^{2}} M_{B}^{4} \sum_{p} h_{B}^{p} \tilde{\partial}_{w^{i}, s^{p}, r^{j}}\left(\hat{L}_{B B}^{(1,1)}(w, s, r)+\hat{L}_{B B}^{(1,2)}(w, s, r)\right) \\
& +\frac{n_{A}}{n} M_{B}^{\frac{5}{2}} M_{A}^{\frac{3}{2}} \sum_{p} h_{A}^{p} \tilde{\partial}_{w^{i}, s^{p}, r^{j}} \hat{L}_{B A}^{(1,1)}-\frac{\pi^{\frac{7}{2}}}{3} \widetilde{\Gamma}\left(\tilde{\partial}_{w^{i}, t^{j}} \hat{H}(w, t)+2 \tilde{\partial}_{w^{i}, t^{j}} \hat{Z}(w, t)\right) \\
& M_{i+(4 N+4), j}^{K}=\frac{\pi^{\frac{7}{2}}}{3} \widetilde{\Gamma} c \frac{\partial \ln \widetilde{\Gamma}}{\partial c} \sum_{q} \tilde{\partial}_{w^{i}, t^{j}} \hat{Z}(w, t)
\end{aligned}
$$




$$
\begin{aligned}
& M_{i+(4 N+4), j+(4 N+4)}^{K}=\frac{n_{A}}{n} \frac{\sigma_{A}^{2}}{\sigma_{A B}^{2}} M_{A}^{4} \sum_{p} h_{A}^{p} \tilde{\partial}_{w^{i}, s^{p}, r^{j}}\left(\hat{L}_{A A}^{(1,1)}(w, s, r)+\hat{L}_{A A}^{(1,2)}(w, s, r)\right) \\
& +\frac{n_{B}}{n} M_{A}^{\frac{5}{2}} M_{B}^{\frac{3}{2}} \sum_{p} h_{B}^{p} \tilde{\partial}_{w^{N}, s^{p}, r^{q}} \hat{L}_{A B}^{(1,1)}(w, s, r) \\
& -\frac{\pi^{\frac{7}{2}}}{3} \widetilde{\Gamma}\left(\tilde{\partial}_{w^{i}, t^{j}} \hat{H}(w, t)+2 \tilde{\partial}_{w^{i}, t^{j}} \hat{Z}(w, t)\right) \\
& M_{i+(4 N+4), j+(5 N+5)}^{K}=\frac{n_{B}}{n} M_{A}^{2} M_{B}^{2} \sum_{p} h_{A}^{p} \tilde{\partial}_{w^{i}, s^{p}, r^{j}} \hat{L}_{A B}^{(1,2)}(w, s, r) \\
& M_{i+(5 N+5), j+(N+1)}^{K}=\frac{\pi^{\frac{7}{2}}}{3} \widetilde{\Gamma} c \frac{\partial \ln \widetilde{\Gamma}}{\partial c} \sum_{q} \tilde{\partial}_{w^{i}, t j} \hat{Z}(w, t) \\
& M_{i+(5 N+5), j+(4 N+4)}^{K}=\frac{n_{A}}{n} M_{B}^{2} M_{A}^{2} \sum_{p} h_{B}^{p} \tilde{\partial}_{w^{i}, s^{p}, r^{j}} \hat{L}_{B A}^{(1,2)}(w, s, r) \\
& M_{i+(5 N+5), j+(5 N+5)}^{K}=\frac{n_{B}}{n} \frac{\sigma_{B}^{2}}{\sigma_{A B}^{2}} M_{B}^{4} \sum_{p} h_{B}^{p} \tilde{\partial}_{w^{i}, s^{p}, r^{j}}\left(\hat{L}_{B B}^{(1,1)}(w, s, r)+\hat{L}_{B B}^{(1,2)}(w, s, r)\right) \\
& +\frac{n_{A}}{n} M_{B}^{\frac{5}{2}} M_{A}^{\frac{3}{2}} \sum_{p} h_{A}^{p} \tilde{\partial}_{w^{N}, s^{p}, r^{q}} \hat{L}_{B A}^{(1,1)}(w, s, r) \\
& -\frac{\pi^{\frac{7}{2}}}{3} \widetilde{\Gamma}\left(\tilde{\partial}_{w^{i}, t^{j}} \hat{H}(w, t)+2 \tilde{\partial}_{w^{i}, t^{j}} \hat{Z}(w, t)\right) \\
& M_{i+(6 N+6), j+(6 N+6)}^{K}=\frac{n_{A}}{n} \frac{\sigma_{A}^{2}}{\sigma_{A B}^{2}} M_{A}^{5} \sum_{p} h_{A}^{p} \tilde{\partial}_{w^{i}, s^{p}, r^{j}}\left(\hat{L}_{A A}^{(2,1)}(w, s, r)+\hat{L}_{A A}^{(2,2)}(w, s, r)\right) \\
& +\frac{n_{B}}{n} M_{A}^{\frac{7}{2}} M_{B}^{\frac{3}{2}} \sum_{p} h_{B}^{p} \tilde{\partial}_{w^{i}, s^{p}, r^{j}} \hat{L}_{A B}^{(2,1)}(w, s, r)-\frac{5 \pi^{\frac{7}{2}}}{6} \widetilde{\Gamma} \tilde{\partial}_{w^{i}, t^{j}} \hat{H}^{V}(w, t) \\
& M_{i+(6 N+6), j+(7 N+7)}^{K}=\frac{n_{B}}{n} M_{A}^{2} M_{B}^{3} \sum_{p} h_{A}^{p} \tilde{\partial}_{w^{i}, s^{p}, r^{j}} \hat{L}_{A B}^{(2,2)}(w, s, r) \\
& M_{i+(7 N+7), j+(6 N+6)}^{K}=\frac{n_{A}}{n} M_{B}^{2} M_{A}^{3} \sum_{p} h_{B}^{p} \tilde{\partial}_{w^{i}, s^{p}, r^{j}} \hat{L}_{B A}^{(2,2)}(w, s, r)
\end{aligned}
$$




$$
\begin{aligned}
M_{i+(7 N+7), j+(7 N+7)}^{K} & =\frac{n_{B}}{n} \frac{\sigma_{B}^{2}}{\sigma_{A B}^{2}} M_{B}^{5} \sum_{p} h_{B}^{p} \tilde{\partial}_{w^{N}, s^{p}, r^{q}}\left(\hat{L}_{B B}^{(2,1)}(w, s, r)+\hat{L}_{B B}^{(2,2)}(w, s, r)\right) \\
& +\frac{n_{A}}{n} M_{B}^{\frac{7}{2}} M_{A}^{\frac{3}{2}} \sum_{p} h_{A}^{p} \tilde{\partial}_{w^{N}, s^{p}, r^{q}} \hat{L}_{B A}^{(2,2)}(w, s, r)-\frac{5 \pi^{\frac{7}{2}}}{6} \widetilde{\Gamma}_{w_{w^{i}, t^{j}}} \hat{H}^{V}(w, t),
\end{aligned}
$$

where the generating functions $\hat{H}^{V}, \hat{H}$, and $\hat{Z}$ are given by:

$$
\begin{aligned}
\hat{H}^{V}(w ; t) & =\frac{\left(-\frac{7}{2} \frac{(1-w)\left(\left(\eta_{2}-1\right)(1-t)+1\right)}{\left(1-w t+\left(\eta_{2}-1\right)(1-t)(1-w)\right)}+3\right)}{\left(1-w t+\left(\eta_{2}-1\right)(1-t)(1-w)\right)^{\frac{7}{2}}} \\
\hat{H}(w ; t) & =-\frac{15}{4} \frac{(1-w)\left(\eta_{2}(1-t)+t\right)}{\left(1-w t+\left(\eta_{2}-1\right)(1-t)(1-w)\right)^{\frac{7}{2}}} \\
\hat{Z}(w ; t) & =\frac{3}{2} \frac{1}{\left(1-w t+\left(\eta_{2}-1\right)(1-t)(1-w)\right)^{\frac{5}{2}}},
\end{aligned}
$$

and the generating functions $\hat{L}_{\alpha \beta}^{(i, j)}(w ; s, r)$ can be computed by taking derivatives of the "supergenerating function", $J_{\alpha \beta}^{(0)}$ (cf. Eq. (B3) in Appendix B):

$$
\begin{aligned}
& \hat{L}_{\alpha \beta}^{(1,1)}(w ; s, r)=\frac{1}{(1-w)^{\frac{5}{2}}(1-r)^{\frac{5}{2}}(1-s)^{\frac{3}{2}}} \\
& \times {\left[\frac{1}{e_{\alpha \beta}^{2}} \delta x J_{\alpha \beta}^{(0)}\left(\frac{w}{1-w} M_{\alpha}, 0, \eta_{2} M_{\alpha}+\frac{r}{1-r} M_{\alpha}, \eta M_{\beta}+\frac{s}{1-s} M_{\beta}, 0,0,0\right)\right.} \\
&\left.-\delta a J_{\alpha \beta}^{(0)}\left(\frac{w}{1-w} M_{\alpha}+\eta_{2} M_{\alpha}+\frac{r}{1-r} M_{\alpha}, \eta M_{\beta}+\frac{s}{1-s} M_{\beta}, 0,0,0,0,0\right)\right] \\
& \hat{L}_{\alpha \beta}^{(1,2)}(w ; s, r)=\frac{1}{(1-w)^{\frac{5}{2}}(1-r)^{\frac{5}{2}}(1-s)^{\frac{3}{2}}} \\
& \times\left[\frac{1}{e_{\alpha \beta}^{2}} \delta y J_{\alpha \beta}^{(0)}\left(\frac{w}{1-w} M_{\alpha}, 0, \eta M_{\alpha}+\frac{s}{1-s} M_{\alpha}, \eta_{2} M_{\beta}+\frac{r}{1-r} M_{\beta}, 0,0,0\right)\right. \\
&\left.\delta z J_{\alpha \beta}^{(0)}\left(\frac{w}{1-w} M_{\alpha}+\eta M_{\alpha}+\frac{s}{1-s} M_{\alpha}, \eta_{2} M_{\beta}+\frac{r}{1-r} M_{\beta}, 0,0,0,0,0\right)\right] \\
& \hat{L}_{\alpha \beta}^{(2,1)}(w ; s, r)=\frac{(\mathrm{A}}{(1-w)^{\frac{5}{2}}(1-r)^{\frac{5}{2}}(1-s)^{\frac{3}{2}}}(\mathrm{~A} 6) \\
& \times\left[\frac{1}{e_{\alpha \beta}^{2}}\left(\delta^{2} x-\frac{1}{3} \delta a \delta c\right) J_{\alpha \beta}^{(0)}\left(\frac{w}{1-w} M_{\alpha}, 0, \eta_{2} M_{\alpha}+\frac{r}{1-r} M_{\alpha}, \eta M_{\beta}+\frac{s}{1-s} M_{\beta}, 0,0,0\right)\right. \\
&\left.-\frac{2}{3} \delta^{2} a J_{\alpha \beta}^{(0)}\left(\frac{w}{1-w} M_{\alpha}+\eta_{2} M_{\alpha}+\frac{r}{1-r} M_{\alpha}, \eta M_{\beta}+\frac{s}{1-s} M_{\beta}, 0,0,0,0,0\right)\right]
\end{aligned}
$$




$$
\begin{aligned}
& \hat{L}_{\alpha \beta}^{(2,2)}(w ; s, r)=\frac{1}{(1-w)^{\frac{5}{2}}(1-r)^{\frac{5}{2}}(1-s)^{\frac{3}{2}}} \\
& \times\left[\frac{1}{e_{\alpha \beta}^{2}}\left(\delta^{2} y-\frac{1}{3} \delta a \delta d\right) J_{\alpha \beta}^{(0)}\left(\frac{w}{1-w} M_{\alpha}, 0, \eta M_{\alpha}+\frac{s}{1-s} M_{\alpha}, \eta_{2} M_{\beta}+\frac{r}{1-r} M_{\beta}, 0,0,0\right)\right. \\
& \left.-\frac{2}{3}\left(\delta^{2} z-\frac{1}{3} \delta a \delta b\right) J_{\alpha \beta}^{(0)}\left(\frac{w}{1-w} M_{\alpha}+\eta M_{\alpha}+\frac{s}{1-s} M_{\alpha}, \eta_{2} M_{\alpha}+\frac{r}{1-r} M_{\beta}, 0,0,0,0,0\right)\right]
\end{aligned}
$$

where $\delta a \equiv-\frac{\partial}{\partial a} ; \delta b \equiv-\frac{\partial}{\partial b} ; \delta c \equiv-\frac{\partial}{\partial c} ; \delta d \equiv-\frac{\partial}{\partial d} ; \delta x \equiv-\frac{\partial}{\partial x}+\frac{1}{2} \delta a+\frac{1}{2} \delta c ; \delta y \equiv-\frac{\partial}{\partial y}+\frac{1}{2} \delta a+\frac{1}{2} \delta d$; $\delta z \equiv-\frac{\partial}{\partial z}+\frac{1}{2} \delta a+\frac{1}{2} \delta b$, and it is understood that the derivatives operators $\delta$ are applied before the substitution of the respective arguments. The vector elements, $R_{i}^{K}$, for $1 \leq i \leq 6 N+6$ are calculated by substituting the expressions (3.24-3.26) into the right hand side of Eq. (3.12), multiplying by the functions $S_{\frac{3}{2}}^{i}\left(\gamma_{\alpha} u^{2}\right) \sqrt{\gamma_{\alpha}} \mathbf{u}=\tilde{\partial}_{w^{i}} G_{\frac{3}{2}}\left(w, \gamma_{\alpha} u^{2}\right) \sqrt{\gamma_{\alpha}} \mathbf{u}$, and integrating over the velocities. The vector elements $R_{i}^{K}$ for $6 N+7 \leq i \leq 8 N+8$ are calculated by substituting the expressions (3.27) into the right hand side of Eq. (3.13), multiplying by the functions $S_{\frac{5}{2}}^{i}\left(\gamma_{\alpha} u^{2}\right) \gamma_{\alpha}^{3 / 2} \overline{\mathbf{u u}}=\tilde{\partial}_{w^{i}} G_{\frac{5}{2}}\left(w, \gamma_{\alpha} u^{2}\right) \gamma_{\alpha}^{3 / 2} \overline{\mathbf{u u}}$, and integrating over the velocities. The results, for $1 \leq i \leq N+1$, are:

$$
\begin{aligned}
R_{i}^{K} & =\frac{\pi^{3}}{\sqrt{M_{A}}} \frac{n_{A}}{\sqrt{\gamma_{A}}} \sum_{p} h_{A}^{p} \widetilde{\partial}_{w^{i}, s^{p}} \hat{R}_{A}^{T}(w ; s) \\
R_{i+(N+1)}^{K} & =\frac{\pi^{3}}{\sqrt{M_{B}}} \frac{n_{B}}{\sqrt{\gamma_{B}}} \sum_{p} h_{B}^{p} \widetilde{\partial}_{w^{N i}, s^{p}} \hat{R}_{B}^{T}(w ; s) \\
R_{i+(2 N+2)}^{K} & =\frac{\pi^{3}}{\sqrt{M_{A}}} \frac{n_{A}}{\sqrt{\gamma_{A}}} \sum_{p} h_{A}^{p} \widetilde{\partial}_{w^{i}, s^{p}} \hat{R}_{A}^{n}(w ; s) \\
R_{i+(3 N+3)}^{K} & =\frac{\pi^{3}}{\sqrt{M_{B}}} \frac{n_{B}}{\sqrt{\gamma_{B}}} \sum_{p} h_{B}^{p} \widetilde{\partial}_{w^{i}, s^{p}} \hat{R}_{B}^{n}(w ; s) \\
R_{i+(4 N+4)}^{K} & =\frac{\pi^{3}}{\sqrt{M_{A}}} \sum_{p}\left(h_{A}^{p}+c \frac{\partial h_{A}^{p}}{\partial c}\right) \widetilde{\partial}_{w^{i}, s^{p}} \hat{R}_{A}^{c}(w ; s) \\
R_{i+(5 N+5)}^{K} & =\frac{\pi^{3}}{\sqrt{M_{B}}} \sum_{p}\left(-\frac{c}{1-c} h_{B}^{p}+c \frac{\partial h_{B}^{p}}{\partial c}\right) \widetilde{\partial}_{w^{i}, s^{p}} \hat{R}_{B}^{c}(w ; s) \\
R_{i+(6 N+6)}^{K} & =\frac{5 \pi^{3}}{\sqrt{M_{A}}} \sum_{p} h_{A}^{p} \widetilde{\partial}_{w^{i}, s^{p}} \hat{R}_{A}^{V}(w, s) \\
R_{i+(7 N+7)}^{K} & =\frac{5 \pi^{3}}{\sqrt{M_{B}}} \sum_{p} h_{B}^{p} \widetilde{\partial}_{w^{i}, s^{p}} \hat{R}_{B}^{V}(w, s)
\end{aligned}
$$


where the generating functions $\hat{R}_{\alpha}^{V}, \hat{R}_{\alpha}^{T}, \hat{R}_{\alpha}^{n}$, and $\hat{R}_{\alpha}^{c}$ are given by:

$$
\begin{gathered}
\hat{R}_{\alpha}^{c}(w ; s)=\frac{3}{2} \frac{(1-s)}{(1-w s+(\eta-1)(1-s)(1-w))^{\frac{5}{2}}} \\
\hat{R}_{\alpha}^{n}(w ; s)=\left(1-\frac{n m_{0}}{\rho} M_{\alpha}\left(\eta+\frac{s}{1-s}\right)\right) \hat{R}_{\alpha}^{c}(w ; s) \\
\hat{R}_{\alpha}^{T}(w ; s)=-\frac{5}{2}\left(1-\frac{\left(\eta+\frac{s}{1-s}\right)}{\left(\frac{w}{1-w}+\frac{s}{1-s}+\eta\right)}\right) \hat{R}_{\alpha}^{c}(w ; s)+\hat{R}_{\alpha}^{n}(w ; s) \\
\hat{R}_{\alpha}^{V}(w ; s)=\frac{(1-s)(\eta(1-s)+s)}{(1-w s+(\eta-1)(1-s)(1-w))^{\frac{7}{2}}}
\end{gathered}
$$

\section{B The super generating function}

Most of the generating functions necessary to derive the constitutive relations can be expressed in terms of derivatives of a "super-generating" function, $J_{\alpha \beta}^{(0)}$, defined by:

$$
J_{\alpha \beta}^{(0)}(a, b, c, d, x, y, z) \equiv \int_{\mathbf{u}_{12} \cdot \mathbf{k}>0} d \mathbf{u}_{1} d \mathbf{u}_{2} d \hat{\mathbf{k}}\left(\mathbf{k} \cdot \mathbf{u}_{12}\right) e^{-F}
$$

where $\hat{\mathbf{k}}$ is a unit vector,

$$
F \equiv a u_{1}^{2}+b u_{2}^{2}+c u_{1}^{\prime 2}+d u_{2}^{\prime 2}+\frac{x}{2}\left(\mathbf{u}_{1}+\mathbf{u}_{1}^{\prime}\right)^{2}+\frac{y}{2}\left(\mathbf{u}_{1}+\mathbf{u}_{2}^{\prime}\right)^{2}+\frac{z}{2}\left(\mathbf{u}_{1}+\mathbf{u}_{2}\right)^{2},
$$

primed vectors denote precollisional velocities, unprimed vectors denotes postcollisional velocities, and the collision law relating the two is given in Eq. (2.1). The indices $\alpha$ and $\beta$ denote the species' identities as explained in the text following Eq. (2.1). The integral can be readily calculated [44] to yield:

$$
J_{\alpha \beta}^{(0)}=\frac{2 \pi^{\frac{7}{2}}}{\lambda^{\frac{3}{2}}} \frac{1}{\mu_{\alpha \beta}\left(\nu_{\alpha \beta}+\mu_{\alpha \beta}\right)}
$$

where $\lambda=a+b+c+d+2 x+2 y+2 z$ and:

$$
\begin{aligned}
\mu_{\alpha \beta} & =R_{\alpha \beta}-\frac{K_{\alpha \beta}^{2}}{\lambda} \\
\nu_{\alpha \beta} & =S_{\alpha \beta}^{2}-\frac{\left(1+e_{\alpha \beta}\right)}{\lambda e_{\alpha \beta}}\left((d+y) M^{\alpha \beta}-(c+x) M^{\beta \alpha}\right) \\
& \times\left(2 K_{\alpha \beta}+\frac{\left(1+e_{\alpha \beta}\right)}{e_{\alpha \beta}}\left((d+y) M^{\alpha \beta}-(c+x) M^{\beta \alpha}\right)\right)
\end{aligned}
$$


with

$$
\begin{aligned}
R_{\alpha \beta} & =(a+c+2 x)\left(M^{\beta \alpha}\right)^{2}+(b+d)\left(M^{\alpha \beta}\right)^{2} \\
& +\frac{1}{2}\left(M^{\alpha \beta}-M^{\beta \alpha}\right)^{2}(y+z) \\
S_{\alpha \beta} & =\frac{1+e_{\alpha \beta}}{2 e_{\alpha \beta}}\left[( \frac { 1 + e _ { \alpha \beta } } { e _ { \alpha \beta } } - 2 ) \left((2 c+x)\left(M^{\beta \alpha}\right)^{2}\right.\right. \\
& \left.\left.+\left(M^{\alpha \beta}\right)^{2}(2 d+y)\right)+2 M^{\alpha \beta} M^{\beta \alpha} y-2\left(M^{\beta \alpha}\right)^{2} x\right] \\
K_{\alpha \beta} & =\left(M^{\beta \alpha}(a+c+2 x+y+z)-M^{\alpha \beta}(b+d+y+z)\right) .
\end{aligned}
$$

\section{References}

[1] B. Ö. Arnarson, J.T. Willits. Thermal diffusion in binary mixtures of smooth, nearly elastic spheres with and without gravity. Phys. Fluids, 10 (1998), No. 1, 1324-1328.

[2] M. Bose, P. R. Nott, V. Kumaran. Excluded-volume attraction in vibrated granular mixtures. Europhys. Lett., 68 (2004), No. 4, 508-514.

[3] J. J. Brey, M. J. Ruiz-Montero, F. Moreno. Hydrodynamics of an open vibrated granular system. Phys. Rev. E, 63 (2001), No. 6, 061305.

[4] J. J. Brey, M. J. Ruiz-Montero, F. Moreno. Energy partition and segregation for an intruder in a vibrated granular system under gravity. Phys. Rev. Lett., 95 (2005), No. 9, 098001.

[5] N. V. Brillantov, T. Pöschel. Breakdown of the Sonine expansion for the velocity distribution of granular gases. Europhys. Lett., 74 (2006), No. 3, 424-430.

[6] R. Brito, H. Enriquez, S. Godoy, R. Soto. Segregation induced by inelasticity in a vibrofluidized granular mixture. Phys. Rev. E, 77 (2008), No. 6, 061301.

[7] D. Brone, F. J. Muzzio. Size segregation in vibrated granular systems: A reversible process. Phys. Rev. E, 56 (1997), No. 1, 1059-1063.

[8] S. Chapman and T. G. Cowling. The mathematical Theory of Nonuniform Gases. Cambridge Univ. Press, London, 1970.

[9] W. Cooke, S. Warr, J. M. Huntley, R. C. Ball. Particle size segregation in a two-dimensional bed undergoing vertical vibration. Phys. Rev. E, 53 (1996), No. 3, 2812-2822.

[10] S. R. de Groot and P. Mazur. Non-Equilibrium Thermodynamics. North-Holland, Amsterdam, 1969.

[11] S. E. Esipov , T. Pöschel. The granular phase diagram. J. Stat. Phys., 86 (1997), No. 5-6, 1385-1395. 
[12] Z. Farkas, F. Szalai, D. E. Wolf, T. Vicsek. Segregation of binary mixtures by a ratchet mechanism. Phys. Rev. E, 65 (2002), No. 2, 022301.

[13] V. Garzó. Segregation in granular binary mixtures: Thermal diffusion. Europhys. Lett., 75 (2006), No. 4, 521-527.

[14] V. Garzó. Brazil-nut effect versus reverse Brazil-nut effect in a moderately dense granular fluid. Phys. Rev. E, 78 (2008), No. 2, 020301.

[15] V. Garzó, J. W. Dufty. Hydrodynamics for a granular binary mixture at low density. Phys Fluids, 14 (2002), No. 4, 1476-14902.

[16] V. Garzó, F. V. Reyes, J. M. Montanero. Modified Sonine approximation for granular binary mixtures. J Fluid Mech., 623 (2009), 387-411.

[17] I. Goldhirsch. Rapid granular flows. Annu Rev Fluid Mech., 35 (2003), 267-293.

[18] I. Goldhirsch, D. Ronis. Theory of thermophoresis I: General considerations and mode coupling analysis. Phys Rev. A, 27 (1983), No. 3, 1616-1634.

[19] I. Goldhirsch, D. Ronis. Theory of thermophoresis II: Low-density behavior. Phys Rev. A, 27 (1983), No. 3, 1635-1656, 1983.

[20] D. C. Hong, P. V. Quinn, S. Luding. The reverse Brazil nut problem: Competition between percolation and condensation. Phys Rev Lett., 86 (2001), No. 15, 3423-3426.

[21] S. S. Hsiau, M. L. Hunt. Granular thermal diffusion in flows of binary-sized mixtures. Acta Mech., 114 (1996), No. 1-4, 121-137.

[22] H. M. Jaeger, S. R. Nagel, and R. P. Behringer. Granular solids, liquids, and gases. Rev Mod Phys., 68 (1996), No. 4, 1259-1273.

[23] J. T. Jenkins, F. Mancini. Kinetic theory for binary mixtures of smooth nearly elastic spheres. Phys Fluids A, 1 (1989), No. 12, 2050-2059.

[24] J. T. Jenkins, D. K. Yoon. Segregation in binary mixture under gravity. Phys Rev Lett., 88 (2002), No. 19, 194304.

[25] J. M. Kincaid, E. G. D. Cohen, M. Lopez de Haro. The Enskog theory for multicomponent mixtures. iv. thermal diffusion. J Chem Phys., 86 (1987), No. 2, 963-975.

[26] J. B. Knight, E. E. Ehrlich, V. Y. Kuperman, J. K. Flint, H. M. Jaeger, S. R. Nagel. Experimental study of granular convection. Phys Rev. E, 54 (1996), No. 5, 5726-5738.

[27] J. B. Knight, H. M. Jaeger, S. R. Nagel. Vibration-induced size separation in granular media, No. 4, The convection connection. Phys Rev Lett., 70 (1993), No. 24, 3728-3731. 
[28] L. Kondic, R. R. Hartley, S. G. K. Tennakoon, B. Painter, R. P. Behringer. Segregation by friction. Europhys Lett., 61 (2003), No. 6, 742-748.

[29] A. Kudrolli. Size separation in vibrated granular matter. Reports on Progress in Physics., 67 (2004), No. 3, 209-247.

[30] L. D. Landau, E. M. Lifshitz. Fluid Mechanics. Pergamon, London, 1959.

[31] M. E. Mobius, X. Cheng, P. Eshuis, S. R. Karczmar, G. S. Nagel, H. M. Jaeger. Effect of air on granular size separation in a vibrated granular bed. Phys Rev. E, 72 (2005), No. 1, 011304.

[32] S. H. Noskowicz, O. Bar-Lev, D. Serero, I. Goldhirsch. Computer-aided kinetic theory and granular gases. Europhys Lett., 79 (2007), No. 6, 60001.

[33] J. M. Ottino, D. V. Khakhar. Mixing and segregation of granular materials. Annu Rev Fluid Mech., 32 (2000), 55-91.

[34] T. Pöschel, N. V. Brillantov, A. Formella. Impact of high-energy tails on granular gas properties. Phys Rev. E, 74 (2006), No. 4, 041302.

[35] T. Pöschel and H. J. Herrmann. Size segregation and convection. Europhys Lett., 29 (1995), No. 2, 123-128.

[36] D. C. Rapaport. Mechanism for granular segregation. Phys Rev. E, 64 (2001), No. 6, 061304.

[37] P. M. Reis, T. Mullin. Granular segregation as a critical phenomenon. Phys Rev Lett., 89 (2002), No. 24, 244301.

[38] A. Rosato, K. J. Strandburg, F. Prinz, R. H. Swendsen. Why the Brazil nuts are on top: size segregation of particulate matter by shaking. Phys Rev Lett., 58 (1987), No. 10, 1038-1040.

[39] M. Schröter, S. Ulrich, J. Keft, J. B. Swift, H. L. Swinney. Mechanism in the size segregation of a binary granular mixture. Phys Rev. E, 74 (2006), No. 1, 011307.

[40] N. Sela and I. Goldhirsch. Hydrodynamic equations for rapid flows of smooth inelastic spheres, to Burnett order. J Fluid Mech., 361 (1998), 41-74.

[41] D. Serero, S. H. Noskowicz, and I. Tan, M. L. Goldhirsch. Layering effects in vertically vibrated systems., Eur. Phys. J. E (2009).

[42] D. Serero. Kinetic Theory of Granular Gas Mixtures. PhD thesis, Tel Aviv University, 2009.

[43] D. Serero, I. Goldhirsch, S. H. Noskowicz, M. L. Tan. Hydrodynamics of granular gases and granular gas mixtures. J Fluid Mech., 554 (2006), 237-258.

[44] D. Serero, S. H. Noskowicz, I. Goldhirsch. Exact versus mean field solutions for granular gas mixtures. Gran. Matt., 10 (2007), No. 1, 37-46. 
[45] T. Shinbrot, F. J. Muzzio. Reverse buoyancy in shaken granular beds. Phys Rev Lett., 81 (1998), No. 20, 4365-4368.

[46] T. Shinbrot, F. J. Muzzio. Nonequilibrium patterns in granular mixing and segregation. "Physics Today", 53 (2000), No. 3, 25-30.

[47] L. Trujillo, M. Alam, H. J. Herrmann. Segregation in a fluidized binary granular mixture: competition between buoyancy and geometric force. Europhys Lett., 64 (2003), No. 2, 190196.

[48] S. Ulrich, M. Schrter, H. L. Swinney. Influence of friction on granular segregation. Phys Rev. E, 76 (2007), No. 4, 042301.

[49] H. Viswanathan, R. D. Wildman, J. M. Huntley, T. W. Martin. Comparison of kinetic theory predictions with experimental results for a vibrated three-dimensional granular bed. Phys Fluids, 18 (2006), No. 11, 113302.

[50] R. D. Wildman, J. T. Jenkins, P. E. Krouskop, J. Talbot. A comparison of the predictions of a simple kinetic theory with experimental and numerical results for a vibrated granular bed consisting of nearly elastic particles. Phys Fluids, 18 (2006), No. 7, 073301.

[51] D. K. Yoon, J. T. Jenkins. The influence of different species' granular temperatures on segregation in a binary mixture of dissipative grains. Phys Fluids, 18 (2006), No. 7, 073303. 